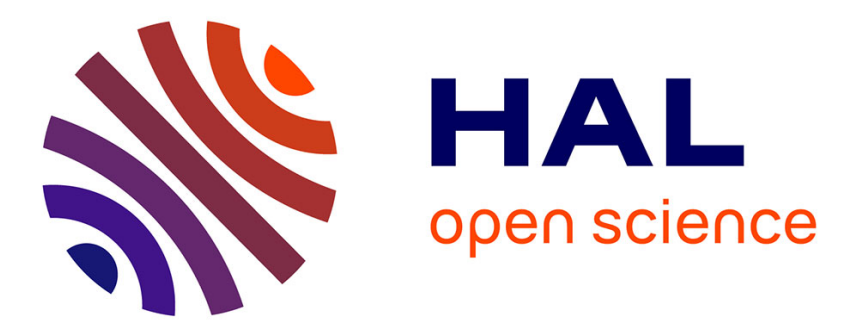

\title{
Fluctuations Internationales et Dynamique du Taux de Change
}

Jean-Olivier Hairault, Thepthida Sopraseuth

\section{To cite this version:}

Jean-Olivier Hairault, Thepthida Sopraseuth. Fluctuations Internationales et Dynamique du Taux de Change. Economie et Prévision, 2008, 2-3 (183-184), pp.65-91. halshs-00270284

\section{HAL Id: halshs-00270284 \\ https://shs.hal.science/halshs-00270284}

Submitted on 4 Apr 2008

HAL is a multi-disciplinary open access archive for the deposit and dissemination of scientific research documents, whether they are published or not. The documents may come from teaching and research institutions in France or abroad, or from public or private research centers.
L'archive ouverte pluridisciplinaire HAL, est destinée au dépôt et à la diffusion de documents scientifiques de niveau recherche, publiés ou non, émanant des établissements d'enseignement et de recherche français ou étrangers, des laboratoires publics ou privés. 


\title{
Fluctuations Internationales et Dynamique du Taux de Change
}

\author{
Jean-Olivier Hairault * Thepthida Sopraseuth ${ }^{\dagger \ddagger}$ \\ Première version : Septembre 2006 \\ Version révisée : Janvier 2006
}

\begin{abstract}
Résumé
Cet article propose une revue de la littérature des MEGIS en économie ouverte. Le MEGIS international de base est incapable de rendre compte de la synchronisation cyclique des PIB observée dans les données, de la faible corrélation croisée des consommations et des fortes variations du taux de change. Ces énigmes empiriques ont donné lieu à une suite d'enrichissements théoriques visant à rendre ces modèles plus conformes aux données. La contribution d'Obstfeld \& Rogoff (1995) s'impose comme un modèle de référence car offrant des fondements microéconomiques à une cadre keynésien en économie ouverte. Les travaux récents tentent de dépasser le modèle néo-keynésien en intégrant des éléments provenant des théories du commerce international ou de choix de portefeuille.
\end{abstract}

Codes JEL : F41, F31

Mots clés : MEGIS, fluctuations internationales, taux de change

\footnotetext{
${ }^{*}$ Université de Paris I, CES, IZA et CEPREMAP. Email : joh@univ-paris1.fr

${ }^{\dagger}$ University d'Evry, EPEE, PSE et CEPREMAP. Email : tsoprase@univ-evry.fr. Adresse : EPEE, Université d'Evry, Département d'Economie, 4 Bd François Mitterrand, 91025 Evry Cedex. Tél : 0169477046 . Fax : 0169477050 .

${ }^{\ddagger}$ Nous remercions le rapporteur anonyme et Jean-Pierre Laffargue pour leurs commentaires ainsi que les participants à la session plénière de l'AFSE (Paris, septembre 2006) consacrée aux MEGIS.
} 


\section{Introduction}

Cet article propose une revue de la littérature consacrée aux fluctuations internationales modélisées dans le cadre de modèles d'équilibre général dynamiques et stochastiques (DSGE). La contribution pionnière de Backus et al. (1994) qui étend le modèle DSGE de base à un cadre international s'est révélée incapable de rendre compte de l'ampleur de la synchronisation cyclique des PIB observée dans les données. Par ailleurs, dans les pays du G7, la corrélation croisée des consommations s'avère faible, observation qui suggère que le partage international des risques est bien moindre que celui inhérent à un modèle international postulant la complétude des marchés financiers. Enfin, le modèle canonique ne rend pas compte de la forte volatilité des taux de change réel et nominal qui caractérisent nos économies depuis la chute du système de Bretton Woods.

Ces énigmes empiriques ont donné lieu à une suite d'enrichissements théoriques visant à rendre ces modèles plus conformes aux données. Ces travaux ont permis une meilleure compréhension de la propagation internationale de chocs asymétriques et des fortes fluctuations du taux de change. La contribution d'Obstfeld \& Rogoff (1995), intégrant concurrence monopolistique et rigidités nominales, s'impose comme un modèle de référence car offrant des fondements microéconomiques à une cadre keynésien en économie ouverte. Les études empiriques viennent valider la pertinence empirique de ce modèle.

Les contributions récentes de la macroéconomie internationale présentent la particularité de renouveler le champ de recherche en liant la macroéconomie ouverte à d'autres aspects connexes tels que le commerce international ou la finance internationale. Nous ne serons pas en mesure de présenter l'ensemble des contributions récentes. Nous choisissons de mettre l'accent sur l'ouverture vers les théories du commerce international. Il s'agit d'intégrer dans des modèles de macroéconomie internationale les enseignements des théories du commerce international concernant les décisions des entreprises sur le choix d'exporter ou de s'implanter sur les marchés étrangers. La structure des flux commerciaux répond de manière endogène aux chocs macroéconomiques, ce qui vient amplifier leurs effets sur les fluctuations du taux de change et la synchronisation cyclique des PIB.

Nous écarterons de cette revue de la littérature les travaux traitant des aspects normatifs. Les articles étudiant la politique monétaire optimale en économie ouverte ou le choix du régime de change ne seront donc pas abordés. Un article de ce numéro spécial est consacré à ce thème.

Nous décrivons en section 2 les faits stylisés internationaux avant de souligner les insuffisances du modèle canonique de Backus et al. (1995) (section 3). Les travaux visant à réduire la distance entre les données et les modèles sont présentés dans la section 4 . La section 5 présente les contributions récentes. 


\section{Les fluctuations internationales : faits stylisés}

Les faits stylisés sont calculés sur les pays du G7 entre 1970 :1 et 1999 :4. L'annexe A fournit une description détaillée des données utilisées. Les séries trimestrielles sont prises en log (à l'exception des exportations nettes qui sont rapportées au PIB) puis filtrées par la méthode de Hodrick \& Prescott (1997). Les statistiques que nous reportons caractérisent le comportement de la composante cyclique. Les caractéristiques des fluctuations nationales sont reportées dans l'annexe B.1.

\subsection{Interdépendances internationales}

Afin de mesurer l'interdépendance à l'échelle internationale, Backus et al. (1995) s'intéressent aux corrélations croisées des productions. Le graphique 1 suggère que les fluctuations des productions aux Etats - Unis, au Canada, en France et plus généralement dans l'Europe des 15 sont effectivement corrélées ${ }^{1}$. En outre, la possibilité de recourir aux marchés financiers internationaux facilite la mutualisation des risques entre les ménages de pays différents et devrait affecter la synchronisation internationale des consommations. Ces deux corrélations constituent deux caractéristiques essentielles dans la compréhension des interdépendances à l'échelle internationale.

Le tableau 1 présente les corrélations contemporaines entre les fluctuations des Etats-Unis et les cycles de chacun de ses partenaires du G7. En ce qui concerne le PIB (noté $Y$ dans le tableau 1) et l'investissement $(I)$, toutes les corrélations sont positives, attestant de la présence de l'interdépendance internationale. Cette conclusion demeure pertinente, excepté pour l'Italie, lorsque nous mesurons la synchronisation des mouvements cycliques de la consommation privée $(C)$ et de l'emploi $(H)$. Les données confirment l'ampleur de l'interdépendance entre les principaux pays industrialisés.

TAB. 1 - Corrélations croisées (1970:1-1999:4)

\begin{tabular}{|l|c|c|c|c|}
\cline { 2 - 5 } \multicolumn{1}{c|}{} & \multicolumn{1}{l}{$\begin{array}{l}\text { Correlation } \\
\text { avec son }\end{array}$} & \multicolumn{1}{l}{$\begin{array}{l}\text { de la variable } \\
\text { homologue }\end{array}$} & \multicolumn{1}{c|}{$\begin{array}{c}\text { de chaque } \\
\text { américain }\end{array}$} & pays \\
\hline Pays & $\mathrm{Y}$ & $\mathrm{C}$ & $\mathrm{I}$ & $\mathrm{H}$ \\
\hline Canada (Can) & 0.60 & 0.79 & 0.36 & 0.55 \\
\hline France (Fra) & 0.27 & 0.35 & 0.15 & 0.25 \\
\hline Allemagne (All) & 0.39 & 0.32 & 0.39 & 0.45 \\
\hline Italie (Ita) & 0.19 & -0.03 & 0.14 & -0.13 \\
\hline Japon (Jap) & 0.31 & 0.35 & 0.35 & 0.05 \\
\hline Royaume Uni (RU) & 0.66 & 0.50 & 0.40 & 0.66 \\
\hline Europe des 15 & 0.48 & 0.42 & 0.38 & 0.29 \\
\hline Médiane & 0.39 & 0.35 & 0.36 & 0.29 \\
\hline
\end{tabular}

\footnotetext{
${ }^{1}$ Nous ne reportons que les fluctuations aux Etats-Unis, au Canada, en France et dans l'Europe des 15 pour plus de clareté.
} 
FIG. 1 - Fluctuations internationales du PIB

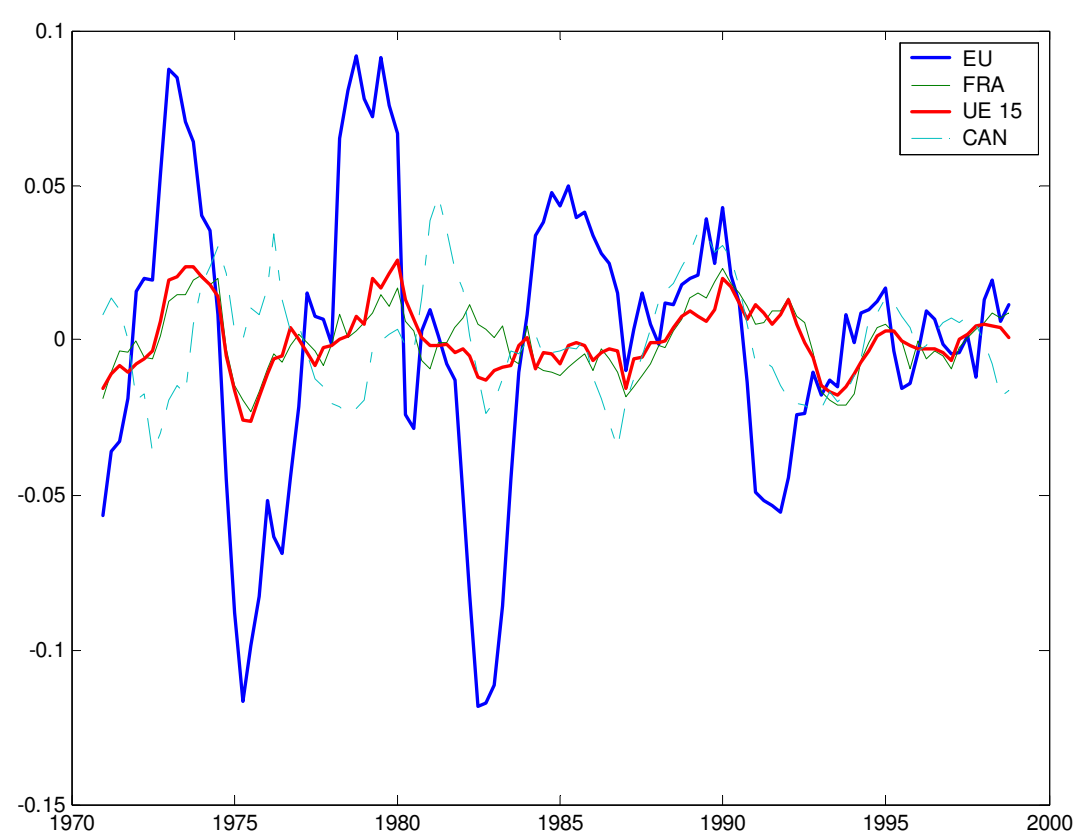

Backus et al. (1995) notent que les consommations sont caractérisées par une synchronisation de moindre ampleur que celle observée pour les productions. Toutefois, le tableau 1 ne leur donne pas tout à fait raison. En effet, sur les huit pays qui composent l'échantillon, trois d'entre eux ne présentent pas cette caractéristique : la corrélation croisée des consommations s'avère supérieure à celle des productions au Canada, en France et au Japon. De plus, dans les autres pays, si les productions sont effectivement davantage corrélées à l'échelle internationale que les consommations privées, l'écart entre les corrélations est faible. Au Royaume Uni, la différence entre corrélations des productions et des consommations est de l'ordre de $0.66-0.50=0.16$, cet écart est bien plus faible en Allemagne $(0.39-0.32=0.07)$ ou pour l'agrégat européen $(0.48-0.42=0.06)$.

L'observation de Backus et al. (1995) constitue-t-elle une observation assez robuste pour devenir un "fait stylisé" que les modèles devront s'attacher à reproduire? Ambler et al. (2004) répondent à cette question par l'affirmative. Ils proposent en effet de mesurer la robustesse de la régularité empirique soulignée par Backus et al. (1995) en ajoutant à leur étude empirique deux éléments : l'élargissement de l'échantillon à 20 pays (soit 190 corrélations croisées) et la mise en oeuvre d'un test fondé sur la méthode des moments généralisés pour juger du caractère significatif de l'écart entre corrélations des productions et des consommations. Ambler et al. (2004) montrent que, dans la période postérieure à 1973, la hiérarchie des corrélations croisées constitue un fait 
stylisé robuste : la corrélation internationale des consommations est significativement moins élevée que celle des productions.

\subsection{Le taux de change}

Pour les tenants de l'approche intertemporelle du solde courant (Sachs (1981), Obstfeld (1982), Frenkel \& Razin (1987)), le taux de change réel reflète les réallocations intertemporelles des ménages. En présence d'un secteur de biens non échangeables, le taux de change réel comprend les prix relatifs des biens échangeables et non échangeables de chaque économie. Cet élément capture les ajustements rendus nécessaires par les déséquilibres extérieurs. Un pays endetté vis-à-vis de l'étranger tentera de résorber son déficit en accentuant sa production et en réduisant sa consommation. Ces réallocations des ressources en faveur du secteur des biens échangeables se traduit par une dépréciation du taux de change réel. De plus, le taux de change réel qui comprend le ratio des prix relatifs internes des biens pourraient refléter les différentiels de productivité entre les secteurs de biens échangeables et non échangeables, comme le soulignent Harrod (1933), Balassa (1964) et Samuelson (1964). Les mouvements du taux de change réel constituent donc un élément essentiel dans l'étude des fluctuations internationales.

Les tableaux 2 et 3 présentent les caractéristiques cycliques du taux de change réel défini comme le prix relatif des paniers de consommation entre les Etats-Unis et chacun de ses partenaires du G7 depuis la fin du système de Bretton Woods ${ }^{2}$. L'annexe A fournit une description détaillée des données utilisées et du calcul du taux de change réel. Le graphique 2 suggère que l'ampleur des fluctuations du taux de change réel est bien supérieure à celle du produit en France. Le tableau 2 confirme que cette observation est robuste : à l'exception du Canada, le rapport des volatilités du taux de change réel $(T C R)$ et du produit avoisine 5 . Il en va de même du taux de change nominal $(T C N)$.

TAB. 2 - Volatilité relative des taux de change (1971:2-1999:4)

\begin{tabular}{|l|c|c|c|}
\hline Pays & $\begin{array}{l}\text { PIB en } \% \\
\sigma_{Y}\end{array}$ & $\begin{array}{c}\text { Taux de change } \\
\text { réel (TCR) } \frac{\sigma_{T C R}}{\sigma_{Y}}\end{array}$ & $\begin{array}{c}\text { Taux de change } \\
\text { nominal (TCN) } \frac{\sigma_{T C N}}{\sigma_{Y}}\end{array}$ \\
\hline Canada & 1.60 & 1.95 & 1.82 \\
\hline France & 1.07 & 7.55 & 8.11 \\
\hline Allemagne & 1.53 & 5.47 & 5.64 \\
\hline Italie & 1.50 & 5.33 & 5.86 \\
\hline Japon & 1.50 & 5.92 & 6.13 \\
\hline Royaume Uni & 1.76 & 4.54 & 4.80 \\
\hline Médiane & 1.51 & 5.40 & 5.75 \\
\hline
\end{tabular}

De plus, le taux de change réel est caractérisé par une forte persistance (tableau 3). Le coefficient d'autocorrélation est de l'ordre de 0.8 dans tous les pays de l'échantillon. La corrélation

\footnotetext{
${ }^{2}$ Les taux de change de tous les pays du G7, à l'exception de l'Italie, deviennent flexibles dès le second trimestre de l'année 1971.
} 
FIG. 2 - Fluctuations du taux de change réel (TCR), du taux de change nominal (TCN) et du PIB en France.

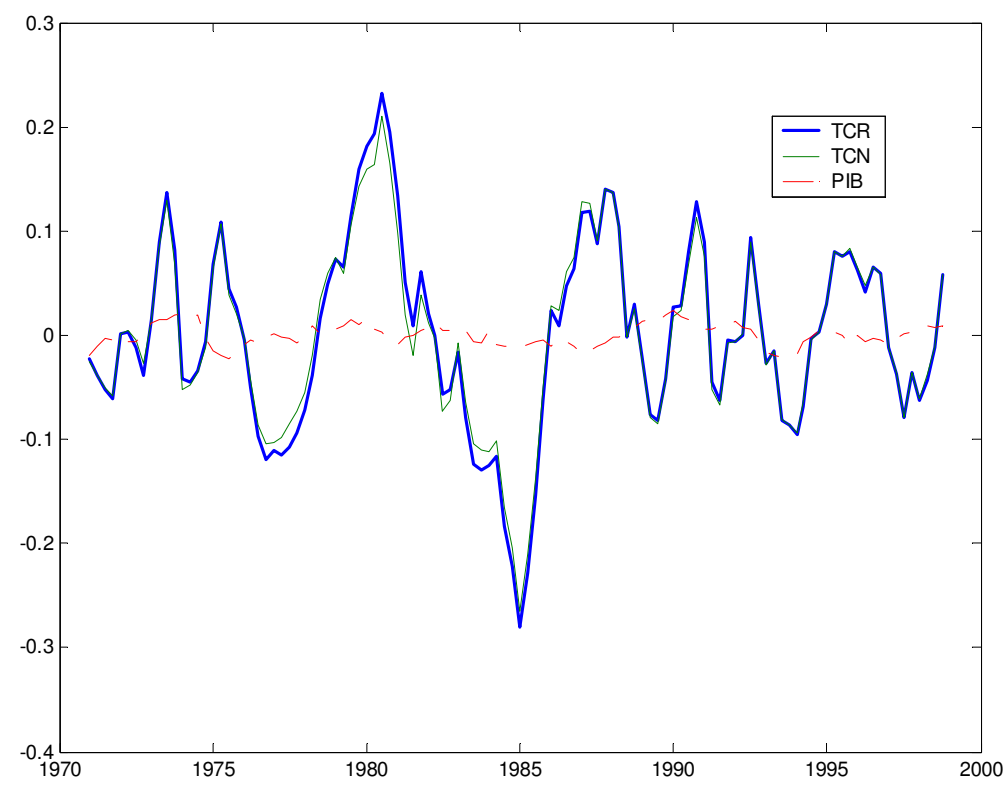

entre le taux de change réel et les exportations nettes (notées $N X$ ) est légèrement positive : l'effet compétitivité qui favorise les exportations dominerait le renchérissement des importations.

TAB. 3 - Corrélations avec le taux de change réel (1971 :2-1999:4)

\begin{tabular}{|l|c|c|c|c|}
\hline & $\begin{array}{l}\text { Persistance du } \\
\text { TCR }\end{array}$ & $\begin{array}{c}\text { Corrélation entre } \\
\text { TCR et TCN }\end{array}$ & $\begin{array}{c}\text { Corrélation entre } \\
\text { TCR et } \mathrm{Y}\end{array}$ & $\begin{array}{c}\text { Corrélation entre } \\
\text { TCR et NX }\end{array}$ \\
\hline Canada & 0.89 & 0.93 & 0.30 & 0.17 \\
\hline France & 0.84 & 0.99 & -0.13 & 0.46 \\
\hline Allemagne & 0.83 & 0.98 & -0.21 & 0.45 \\
\hline Italie & 0.83 & 0.98 & -0.28 & 0.41 \\
\hline Japon & 0.77 & 0.91 & 0.20 & 0.32 \\
\hline Royaume Uni & 0.81 & 0.97 & -0.07 & -0.04 \\
\hline Médiane & 0.83 & 0.98 & -0.10 & 0.36 \\
\hline
\end{tabular}

Par ailleurs, les statistiques reportées dans le tableau 3 nous fournissent des indications quant aux déterminants de la dynamique du taux de change réel. La corrélation contemporaine entre les fluctuations des taux de change réel et nominal atteint plus de 0.9 dans tous les pays considérés. Ce point souligné par Mussa (1986) corrobore les travaux de Isard (1977) et Giovannini (1988) et suggère l'existence de rigidités nominales. En effet, le taux de change réel est composé du taux de change nominal et du prix relatif de l'ensemble des biens. La forte corrélation entre 
taux de change nominaux et réels laisse à penser que les mouvements des prix relatifs jouent un rôle mineur dans les fluctuations du taux de change réel. Les rigidités de prix pourraient être à l'origine de ce phénomène. Ces dernières seront au coeur du modèle d'Obstfeld \& Rogoff (1995) évoqué dans la section 4.3.

\section{Les limites du modèle international canonique}

Les faits stylisés identifiés dans la section précédente constituent les caractéristiques cycliques essentielles dans la compréhension des fluctuations internationales. L'interdépendance entre les principaux pays industrialisés constitue un phénomène robuste. La synchronisation cyclique est manifeste pour la production, la consommation, l'investissement et les heures travaillées. En outre, les corrélations croisées des productions sont plus prononcées que celles des consommations. Enfin, les taux de change réel comme les termes de l'échange sont caractérisés par des fluctuations persistantes et plus volatiles que celles du produit.

Le modèle canonique présenté dans cette section comprend deux pays et deux biens, chaque pays étant spécialisé dans la production d'un bien. Ce modèle s'apparente à celui développé par Backus et al. (1994). Nous adaptons la formulation de Backus et al. (1994) à une spécification homogène avec la présentation des modèles internationaux de cet article. En particulier, au lieu d'utiliser une fonction d'Armington pour agréger les biens à la manière de Backus et al. (1994), nous définissons un bien composite pour la consommation et l'investissement. Ces modifications ne sont pas de nature à affecter significativement les mécanismes du modèle de Backus et al. (1994).

\subsection{Présentation du modèle}

Le modèle est constitué de deux pays. Chaque pays est spécialisé dans la production d'un bien. Chaque économie est peuplée d'une multitude d'agents identiques dont la durée de vie est infinie. Nous considérons donc un ménage représentatif par pays. Le capital est mobile à l'échelle internationale tandis que le travail doit demeurer en deçà des frontières nationales.

\subsubsection{Le ménage}

Chaque pays (pays 1 ou pays national, pays 2 ou pays étranger) est spécialisé dans la production d'un bien (bien 1 et 2). Le ménage représentatif du pays $i$ maximise

$$
E_{0} \sum_{t=0}^{\infty} \beta^{t} U_{i t}\left(C_{i t}^{C}, H_{i t}\right)
$$

où $C_{i t}^{C}$ désigne la quantité de bien composite consommée et $H_{i t}$ les heures travaillées. L'utilité instantanée est 


$$
U_{i t}\left(C_{i t}^{C}, L_{i t}\right)=\log \left(C_{i t}^{C}\right)+\gamma_{H} \ln \left(1-H_{i t}\right)
$$

lorsque le temps disponible est normalisé à l'unité. L'agent représentatif du pays $i$ consomme un panier CES des deux biens.

$$
C_{i}^{C}=\left[\gamma^{\frac{1}{\theta}} C_{i}^{\frac{\theta-1}{\theta}}+(1-\gamma)^{\frac{1}{\theta}} C_{j \neq i}^{\frac{\theta-1}{\theta}}\right]^{\frac{\theta}{\theta-1}} \quad \text { pour } i, j=1,2
$$

Le consommateur du pays $i$ consomme des biens locaux (en quantité $C_{i}$ ) comme étrangers (en quantité $\left.C_{j}\right)$. $(1-\gamma)$ constitue la part du bien étranger dans le panier de consommation. On note $\theta$ l'élasticité de substitution entre les biens nationaux et étrangers. L'indice de prix à la consommation est

$$
P_{i}^{C}=\left[\gamma P_{i}^{1-\theta}+(1-\gamma) P_{j \neq i}^{1-\theta}\right]^{\frac{1}{1-\theta}} \quad \text { pour } i=1,2
$$

où $P_{i}$ est le prix du bien $i$. Le prix du bien 1 est normalisé à l'unité. Soit $\tau$ le prix (relatif) du bien étranger $\frac{P_{2}}{P_{1}} \cdot \tau$ correspond donc aux termes de l'échange (prix des importations rapporté au prix des exportations). Le taux de change réel $\Gamma$ mesure en revanche le prix relatif des paniers de consommation $\frac{P_{2}^{C}}{P_{1}^{C}}$.

Lorsque le ménage consomme autant de bien local qu'étranger $(\gamma=0.5)$, l'équation conduit à $P_{2}^{C}=P_{1}^{C}$. Le taux de change réel $\Gamma$ est alors unitaire. A l'inverse, dès lors que $\gamma \neq 0.5$, d'après $(2), P_{2}^{C} \neq P_{1}^{C}$. L'asymétrie des préférences des agents rend la parité des pouvoirs d'achat caduque de sorte que le taux de change réel n'est plus constant.

Dans la section 3.2, le paramètre $\gamma$ est étalonné à une valeur supérieur à 0.5 traduisant une préférence pour les biens nationaux. Dans la section 5.2, nous présenterons des articles qui expliquent cette préférence nationale à l'aide des coûts de transport.

Dans le modèle log-linéarisé autour de l'état stationnaire,

$$
\hat{\Gamma}_{t}=(2 \gamma-1) \hat{\tau}_{t}
$$

où les variables sont exprimées en écart relatif à leurs valeurs d'état stationnaire. A l'exception de la volatilité, les propriétés cycliques du taux de change réel $\Gamma$ et des termes de l'échange $\tau$ sont donc similaires. Nous retrouvons pour $\gamma=0.5$, la constance du taux de change réel, soit $\hat{\Gamma}_{t}=0$.

Les marchés financiers sont complets. A chaque état de la nature $s_{t+1}$ est associé un titre contingent qui, acheté par le ménage en $t$ au prix $\chi\left(s_{t+1}\right)$, constitue une promesse de verser en $t+1$ une unité de bien 1 si l'état de la nature se réalise en $t+1$. Les ressources de l'agent du pays $i$ sont constituées des revenus associés à sa détention de titres contingents $B_{i}\left(s_{t}\right)$ et à son travail $w_{i t} H_{i t}$ où le salaire horaire $w_{i t}$ est versé en bien $i$. La ménage $i$ utilise ses ressources pour consommer et modifier son portefeuille de titres. 
La contrainte budgétaire de l'agent du pays 1 s'écrit en termes de biens 1

$$
P_{1 t}^{C} C_{1 t}^{C}+\int \chi\left(s_{t+1}\right) B_{1}\left(s_{t+1}\right) d s_{t+1} \leq B_{1}\left(s_{t}\right)+w_{1 t} H_{1 t}
$$

La contrainte budgétaire de son homologue étranger s'écrit, toujours en unités de biens 1

$$
P_{2 t}^{C} C_{2 t}^{C}+\int \chi\left(s_{t+1}\right) B_{2}\left(s_{t+1}\right) d s_{t+1} \leq B_{2}\left(s_{t}\right)+w_{2 t} H_{2 t} \tau_{t}
$$

Soient $\lambda_{1 t}$ et $\lambda_{2 t}$ les multiplicateurs associés aux contraintes budgétaires des ménages 1 et 2. Chaque ménage maximise (1) sous sa contrainte budgétaire. En notant $f\left(s_{t+1}, s_{t}\right)$ la fonction de densité décrivant le passage de l'état de la nature $s_{t+1}$ à l'état de la nature $s_{t}$, l'écriture sous forme récursive du programme du ménage du pays $i$ conduit à

$$
\begin{aligned}
& V\left[B_{i}\left(s_{t}\right)\right]= \\
& \quad \underset{C_{i t}^{C}, H_{i t}, B_{i}\left(s_{t+1}\right)}{\operatorname{Max}}\left\{\begin{array}{c}
U\left(C_{i t}^{C}, L_{i t}\right) \\
+\beta \int\left[B_{i}\left(s_{t+1}\right)\right] f\left(s_{t+1}, s_{t}\right) d s_{t+1}
\end{array}\right\}
\end{aligned}
$$

sous la contrainte budgétaire. Les choix optimaux de la consommation et des titres contingents obéissent à

$$
\begin{array}{rlrl}
\chi\left(s_{t+1}\right) \lambda_{i t} & =\beta \lambda_{i t+1} f\left(s_{t+1}, s_{t}\right) & i=1,2 \\
\frac{\partial U\left(C_{i t}^{C}, L_{i t}\right)}{\partial C_{i t}^{C}} & =\frac{1}{C_{i t}^{C}}=\lambda_{i t} P_{i t}^{C} & i=1,2
\end{array}
$$

tandis que le choix de loisir est tel que

$$
\begin{aligned}
\frac{\gamma_{H}}{1-H_{1 t}} & =\lambda_{1 t} w_{1 t} \\
\frac{\gamma_{H}}{1-H_{2 t}} & =\lambda_{2 t} w_{2 t} \tau_{t}
\end{aligned}
$$

\subsubsection{L'entreprise}

La technologie, décrite par une fonction Cobb-Douglas, est identique dans chaque pays

$$
Y_{i t}=a_{i t}\left(K_{i t}^{C}\right)^{\alpha}\left(H_{i t}\right)^{1-\alpha} \quad i=1,2
$$

où $K_{i t}$ désigne le stock de capital dans le pays $i$. Les chocs technologiques exogènes $a_{1 t}$ et $a_{2 t}$ sont spécifiques à chaque pays et suivent le processus stochastique suivant

$$
\begin{aligned}
\left(\begin{array}{l}
\log \left(a_{1 t}\right) \\
\log \left(a_{2 t}\right)
\end{array}\right)= & \left(\begin{array}{ll}
\rho_{a 1} & \rho_{12}^{a} \\
\rho_{12}^{a} & \rho_{a 2}
\end{array}\right)\left(\begin{array}{l}
\log \left(a_{1 t-1}\right) \\
\log \left(a_{2 t-1}\right)
\end{array}\right)+\left(\begin{array}{cc}
1-\rho_{a, 1} & -\rho_{12}^{a} \\
-\rho_{12}^{a} & 1-\rho_{a, 2}
\end{array}\right)\left(\begin{array}{l}
\log \left(a_{1}\right) \\
\log \left(a_{2}\right)
\end{array}\right) \\
& +\left(\begin{array}{cc}
1 & \psi \\
\psi & 1
\end{array}\right)\left(\begin{array}{c}
\varepsilon_{1 t}^{a} \\
\varepsilon_{2 t}^{a}
\end{array}\right)
\end{aligned}
$$

avec $a_{i}$ l'espérance du choc de productivité $a_{i t},\left|\rho_{a 1}\right|<1$ et $\left|\rho_{a 2}\right|<1$. $\rho_{12}^{a}$ constitue le paramètre de diffusion internationale des impulsions technologiques. Les innovations des chocs 
technologiques, $\varepsilon_{1}^{a}$ et $\varepsilon_{2}^{a}$, ne sont pas corrélées temporellement. De plus, $E\left(\varepsilon_{i t}^{a}\right)=0 \quad \forall i=1,2$. Le paramètre $\psi$ règle l'ampleur de la transmission instantanée des chocs de productivité ${ }^{3}$. Pour $\psi=0$, les chocs sont asymétriques.

On suppose l'existence de coûts associés à l'accumulation du capital

$$
C A_{i t}=\frac{\phi}{2}\left(K_{i t+1}^{C}-K_{i t}^{C}\right)^{2}
$$

L'investissement, à l'instar de la consommation, provient de l'agrégation des deux biens

$$
I_{i}^{C}=\left[\gamma^{\frac{1}{\theta}} I_{i}^{\frac{\theta-1}{\theta}}+(1-\gamma)^{\frac{1}{\theta}} I_{j \neq i}^{\frac{\theta-1}{\theta}}\right]^{\frac{\theta}{\theta-1}} \quad \text { pour } i=1,2
$$

L'évolution du stock de capital obéit à

$$
K_{i t+1}^{C}=(1-\delta) K_{i t}^{C}+I_{i t}^{C}
$$

Le programme des firmes est donc dynamique. Les firmes du pays 1 maximisent

$$
E_{0} \sum_{t=0}^{\infty} \sum_{s_{t+1}} \chi\left(s_{t+1}\right)\left(Y_{1 t}-C A_{1 t}-P_{1 t}^{C} I_{1 t}^{C}-w_{1 t} H_{1 t}\right)
$$

sous la contrainte (10) dont le multiplicateur est $q_{1 t}$. Symétriquement, la firme du pays 2 maximise

$$
E_{0} \sum_{t=0}^{\infty} \sum_{s_{t+1}} \chi\left(s_{t+1}\right)\left[\tau_{t}\left(Y_{2 t}-C A_{2 t}-w_{2 t} H_{2 t}\right)-P_{2 t}^{C} I_{2 t}^{C}\right]
$$

sous la contrainte (10) dont le multiplicateur est $q_{2 t}$. La demande de travail est dictée par

$$
w_{i t}=(1-\alpha) \frac{Y_{i t}}{H_{i t}} \quad i=1,2
$$

tandis que la demande de capital dans le pays 1 obéit à

$$
\begin{aligned}
q_{1 t} & =P_{1 t}^{C}+\phi\left(I_{1 t}^{C}-\delta K_{1 t}^{C}\right) \\
q_{1 t} & =\beta E_{t}\left[\frac{\lambda_{1 t+1}}{\lambda_{1 t}}\left(\alpha \frac{Y_{1 t+1}}{K_{1 t+1}^{C}}+q_{1 t+1}-\delta P_{1 t+1}^{C}\right)\right]
\end{aligned}
$$

Symétriquement, dans le pays 2 , on a

$$
\begin{aligned}
q_{2 t} & =P_{2 t}^{C}+\tau_{t} \phi\left(I_{2 t}^{C}-\delta K_{2 t}^{C}\right) \\
q_{2 t} & =\beta E_{t}\left[\frac{\lambda_{2 t+1}}{\lambda_{2 t}}\left(\tau_{t+1} \alpha \frac{Y_{2 t+1}}{K_{2 t+1}^{C}}+q_{2 t+1}-\delta P_{2 t+1}^{C}\right)\right]
\end{aligned}
$$

\footnotetext{
${ }^{3}$ La corrélation instantanée des innovations des chocs technologiques est

$$
\rho\left(\varepsilon_{1 t}^{a}, \varepsilon_{2 t}^{a}\right)=\frac{2 \psi}{1+\psi^{2}}
$$
}


La contrainte mondiale est en termes de bien 1

$$
Y_{1 t}+\tau_{t} Y_{2 t}=\sum_{i=1}^{2} P_{i}^{C}\left(C_{i t}^{C}+I_{i t}^{C}\right)+C A_{1 t}+\tau_{t} C A_{2 t}
$$

tandis que l'équilibre sur le marché des titres contingents se traduit par

$$
B_{1}\left(s_{t}\right)+B_{2}\left(s_{t}\right)=0 \quad \forall s_{t}
$$

\subsection{Etalonnage}

L'étalonnage correspond à une fréquence trimestrielle. Les Etats-Unis constituent le pays de référence. L'étalonnage des deux pays est symétrique. $\gamma_{H}$ est calculé sachant que $20 \%$ de la période est consacré au travail. $\beta$, le facteur d'escompte psychologique, correspond à un taux d'escompte de $1.2 \%$ par trimestre soit une valeur de 0.988. Depuis 1945, le rapport des revenus salariaux totaux au PNB nord-américain s'établit à 0.58 en moyenne, ce qui équivaut à $\alpha=0.42$. En affectant au taux de dépréciation du capital une valeur de $10 \%$ par an, $\delta$ s'élève à $2.5 \%$ par trimestre. Selon les estimations de Craine (1975), le facteur d'échelle des coûts d'ajustement sur le capital $\phi$ s'élèverait à 0.05. A l'instar de Backus et al. (1995), nous étalonnons l'élasticité de substitution entre les biens à $\theta=1.5$. $\gamma$ la part des biens nationaux dans la consommation et l'investissement est étalonné à 0.8 (Kollman (1996)).

Le choc technologique affecte la productivité globale du modèle. Dans un modèle parfaitement concurrentiel et en présence de rendements d'échelle constants, le résidu de Solow (1957) constitue une mesure du progrès technique. Solow (1957) considère le progrès technologique comme le taux de croissance du produit qui n'est pas expliqué par la croissance des facteurs. L'expression du résidu de Solow est donc

$$
\Delta R S_{t}=\Delta Y_{t}-F_{K, t} \frac{K_{t}}{Y_{t}} \Delta \log \left(K_{t}\right)-F_{H, t} \frac{H_{t}}{Y_{t}} \Delta \log \left(H_{t}\right)
$$

L'hypothèse de concurrence pure et parfaite implique

$$
\begin{aligned}
& w_{t}=F_{H, t} \\
& r_{t}=F_{K, t}
\end{aligned}
$$

soit

$$
\Delta R S_{t}=\Delta Y_{t}-r_{t} \frac{K_{t}}{Y_{t}} \Delta \log \left(K_{t}\right)-w_{t} \frac{H_{t}}{Y_{t}} \Delta \log \left(H_{t}\right)
$$

L'existence de rendements d'échelle constants nous permet d'écrire

$$
\Delta R S_{t}=\Delta Y_{t}-\alpha \Delta \log \left(K_{t}\right)-(1-\alpha) \Delta \log \left(H_{t}\right)
$$

Backus et al. (1992) considèrent que les ajustement du capital prennent du temps et sont donc peu susceptibles de jouer un rôle essentiel dans les mouvements du résidu de Solow. Backus 
et al. (1992) espèrent ainsi échapper aux critiques afférentes aux erreurs de mesure du stock de capital physique. L'expression des résidus de Solow devient

$$
\Delta R S_{t}=\Delta Y_{t}-(1-\alpha) \Delta \log \left(H_{t}\right)
$$

L'estimation du processus suivi par le résidu de Solow, purgé de sa croissance déterministe, conduit à

$$
\log \left(R S_{t}\right)=(1-\rho) \log (R S)+\rho \log \left(R S_{t-1}\right)+\varepsilon_{t}
$$

Puisque le modèle comprend deux pays de taille identique, il s'agit d'estimer le processus joint de deux résidus de Solow. Backus et al. (1992) effectuent cette estimation sur la base des résidus de Solow des Etats-Unis et d'un agrégat Européen entre $1970: 1$ et $1986: 4$ et retiennent un étalonnage symétrique tel que $\rho_{a}=0.906$ et $\rho_{12}^{a}=0.088$. Le tableau 4 résume l'étalonnage.

TAB. 4 - Etalonnage du modèle à deux pays

\begin{tabular}{|c|c|c|c|c|c|c|c|c|c|c|}
\hline$\alpha$ & $\beta$ & $H$ & $\varphi$ & $\delta$ & $\theta$ & $\rho_{a}$ & $\rho_{12}^{a}$ & $\psi$ & $\sigma_{\varepsilon^{a}}$ & $\gamma$ \\
\hline 0.42 & 0.988 & 0.2 & 0.05 & 0.025 & 1.5 & 0.906 & 0.088 & 0.13 & 0.00852 & 0.8 \\
\hline
\end{tabular}

La méthode de Farmer (1993) est utilisée pour résoudre le modèle. L'ensemble des conditions du premier ordre et des équations d'équilibre sur les marchés constituent un système dynamique non linéaire. Nous procédons à une log-linéarisation du système autour de cet état stationnaire avant de déterminer les règles de décisions. L'annexe $\mathrm{C}$ décrit la procédure de résolution et de simulation.

\subsection{Le modèle ne parvient pas à expliquer les corrélations internationales des variables agrégées}

Nous insistons dans cette section sur les caractéristiques internationales qui constituent l'objet de cet article. Les caractéristiques cycliques nationales sont reportées et commentées dans l'Annexe B.2.

Comme l'atteste le tableau 5, le modèle canonique implique des corrélations croisées qui demeurent bien inférieures à celles observées dans la période 1970 :1-1999 :4. La corrélation internationale des productions calculée par simulation stochastique du modèle s'élève à 0.10 alors que la médiane observée sur l'ensemble des pays du G7 est de l'ordre de 0.40. Il en va de même des heures travaillées. L'investissement connaît des évolutions asymétriques dans le modèle avec une corrélation croisée de - 0.13 tandis que les faits stylisés mettent en évidence une covariation internationale positive de cette variable.

L'examen des réponses du système dynamique à un choc de productivité nous éclaire sur les raisons de l'incapacité du modèle à fournir une représentation satisfaisante de l'interdépendance. Le graphique 3 présente les fonctions de réponse à la suite d'une augmentation temporaire de 
TAB. 5 - Interdépendance

\begin{tabular}{|c|c|c|}
\hline & Modèle canonique & Faits stylisés (Médiane) \\
\hline$\rho\left(Y_{1}, Y_{2}\right)$ & 0.10 & 0.39 \\
\hline$\rho\left(H_{1}, H_{2}\right)$ & 0.07 & 0.29 \\
\hline$\rho\left(I_{1}^{C}, I_{2}^{C}\right)$ & -0.13 & 0.36 \\
\hline
\end{tabular}

FIG. 3 - Réponses à un choc technologique asymétrique dans le pays 1
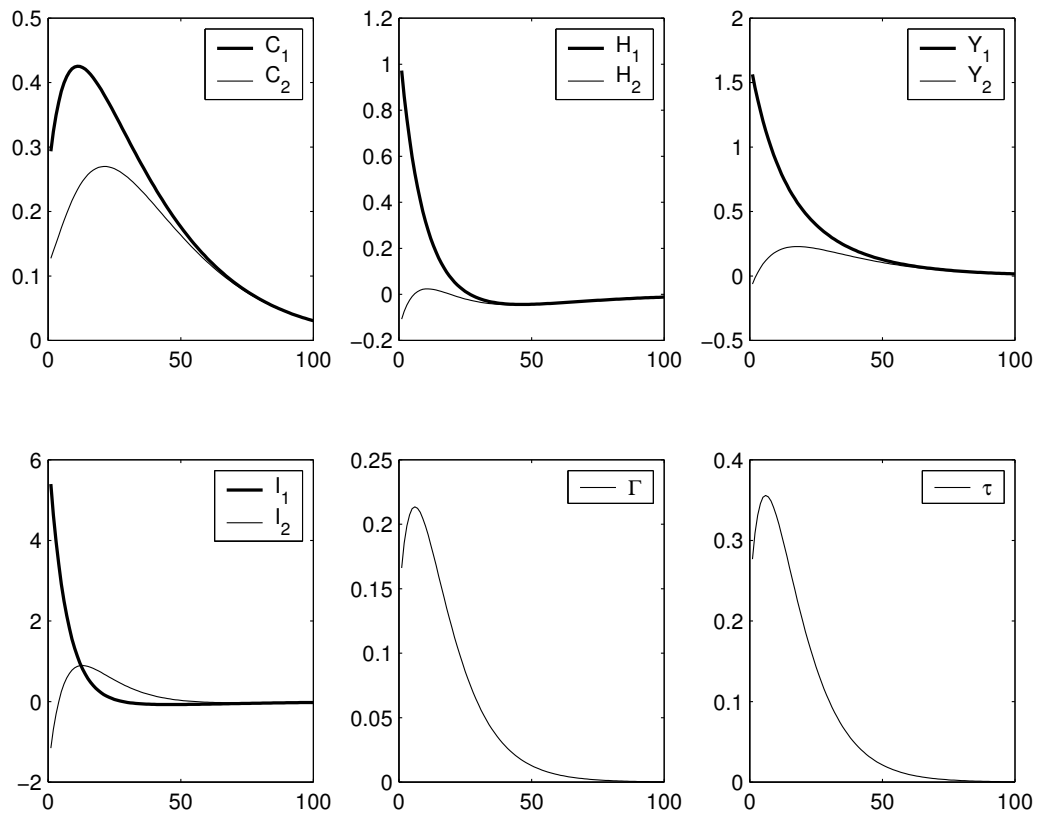

$1 \%$ de la productivité globale dans le pays 1 . Afin d'identifier les mécanismes inhérents au modèle, nous considérons un choc asymétrique qui n'est pas transmis instantanément à l'étranger $(\psi=0)$. Les variables sont exprimées en écart relatif à leur état stationnaire.

Les trajectoires illustrent l'évolution contrastée des productions, des niveaux d'investissement et des heures travaillées. Notons que la faible interdépendance prédite par le modèle tient à la réponse instantanée asymétrique des variables nationales et étrangères. Dans la période du choc, l'écart entre les productions des deux pays se traduit par des dynamiques opposées le long de la trajectoire d'ajustement vers l'état stationnaire.

A la période du choc : L'examen des conditions du premier ordre fournissent les intuitions qui expliquent les insuffisances du modèle. En reprenant l'expression de la fonction de production (8), le stock de capital étant prédéterminé, la réponse instantanée des productions provient des mouvements des chocs technologiques et des heures travaillées. Il convient donc d'expliquer le saut de l'emploi dans les deux pays pour comprendre les mouvements des productions.

La condition du premier ordre relative au choix de portefeuille d'un titre contingent $b_{i}\left(s_{t+1}\right)$ 
(équation (4)) conduit dans les deux pays à

$$
\frac{\lambda_{1 t+1}}{\lambda_{1 t}}=\frac{\lambda_{2 t+1}}{\lambda_{2 t}}=\Omega
$$

Le rapport des utilités marginales associées à la richesse est constant et égal à $\Omega$. En faisant l'hypothèse que la répartition initiale de la richesse entre les deux pays est égalitaire $(\Omega=1)$, on a

$$
\lambda_{1 t}=\lambda_{2 t}
$$

En remplaçant dans (16) les $\lambda_{i t}$ par leurs expressions données par le choix de loisir (équations (6) et (7)) puis en tenant compte que les salaires sont égaux à la productivité marginale du travail (11), on a

$$
\left(\frac{\gamma_{H}}{1-H_{1 t}}\right) \frac{1}{(1-\alpha) a_{1 t} K_{1 t}^{\alpha} H_{1 t}^{-\alpha}}=\left(\frac{\gamma_{H}}{1-H_{2 t}}\right) \frac{1}{(1-\alpha) a_{2 t} K_{2 t}^{\alpha} H_{2 t}^{-\alpha} \tau_{t}}
$$

Tout d'abord, un choc technologique favorable au pays 1 détériore les termes de l'échange dans le pays 1 puisque le choc d'offre positif conduit à une baisse du prix du bien national de sorte que $\tau_{t}$ s'accroît.

L'expansion de la productivité globale $a_{1 t}$ dans le pays 1 incite le ménage à augmenter son offre de travail par un effet de substitution intertemporelle ( $H_{1 t}$ augmente). Toutefois, l'effet direct du choc technologique favorable $\left(a_{1 t}\right)$ qui fait baisser le terme de gauche de l'équation (17) domine l'effet indirect de $H_{1 t}$ qui le fait augmenter.

Pour que l'expression (17) demeure vérifiée, il faut que le terme de droite diminue : la productivité marginale du travail $\left((1-\alpha) a_{2 t} K_{2 t}^{\alpha} H_{2 t}^{-\alpha}\right)$ doit augmenter plus que sa désutilité marginale $\left(\frac{\gamma_{H}}{1-H_{2 t}}\right)$. Dans le cas d'un choc asymétrique, $a_{2 t}$ n'est pas modifié tandis que $K_{2 t}$, le stock de capital, est prédéterminé. En conséquence, les heures travaillées à l'étranger $H_{2 t}$ doivent baisser. Cet effet est atténué par la détérioration des termes de l'échange $\tau_{t}$. La corrélation négative de l'emploi est à l'origine de l'évolution contrastée des produits. En un mot, grâce à la complétude des marchés financiers, le choc de productivité $a_{1 t}$ engendre un effet richesse positif pour le ménage 1 comme le ménage 2 . Ce dernier réduit donc son offre de travail, ce qui engendre une baisse instantanée de la production.

Dynamique agrégée après le choc : Les productions et les heures travaillées dans les deux pays répondent instantanément de manière asymétrique au choc technologique dans le pays 1. Après la période initiale du choc, les évolutions des productions et des heures travaillées demeurent contrastées. En effet, le choc de productivité asymétrique provoque instantanément une fuite des capitaux vers le pays qui offre la rémunération la plus élevée, et ce jusqu'à ce que les flux de capitaux assurent l'égalisation des productivités marginales du capital. Même en présence de frictions dans les flux commerciaux, ce mouvement asymétrique de l'investissement 
provoque des évolutions contrastées des stocks de capital. Cela n'est pas sans conséquence sur les productivités du travail. Le pays qui voit son stock de capital s'accroître profite d'une hausse de sa productivité du travail. Le pays voisin subit une influence inverse. Ainsi, la corrélation négative de l'investissement aujourd'hui explique une corrélation négative de l'emploi demain. Sous l'effet de ces deux évolutions contrastées, le modèle implique une corrélation négative des produits le long de la trajectoire d'ajustement.

La corrélation négative des niveaux de production est obtenue pour un choc dans un seul pays. Quand les chocs ont lieu dans les deux pays, comme c'est le cas dans les simulations stochastiques, cette corrélation devient positive, mais demeure inférieure à celle observée dans les données. Le modèle fournit une mauvaise représentation de l'interdépendance de la production, de l'investissement et de l'emploi. A cette défaillance du modèle s'ajoute un second échec : le modèle ne parvient pas à reproduire l'écart-type du taux de change réel qui demeure trop faible par rapport aux faits stylisés, ni la corrélation croisée des consommations trop élevée pour être conforme à l'observation. Ce point est développé dans la section suivante.

\subsection{Le modèle ne parvient pas à expliquer la corrélation internationale de la consommation, ni la forte volatilité du taux de change réel}

Le tableau 6 illustre les mauvaises performances du modèle concernant l'interdépendance de la consommation et la volatilité des prix relatifs internationaux. La corrélation croisée des consommation est de l'ordre de 0.80 , soit un niveau deux fois supérieur à celui observé (0.35). De plus, les volatilité relative des termes de l'échange et du taux de change réel s'avèrent bien insuffisantes au regard des faits stylisés.

TAB. 6 - Corrélation croisée de la consommation et volatilité du taux de change réel

\begin{tabular}{|c|c|c|}
\hline & Modèle & Faits stylisés (Mediane) \\
\hline$\rho\left(C_{1}^{C}, C_{2}^{C}\right)$ & 0.79 & 0.35 \\
\hline$\frac{\sigma_{\tau}}{\sigma_{Y}}$ & 0.32 & 3.50 \\
\hline$\frac{\sigma_{\Gamma}}{\sigma_{Y}}$ & 0.18 & 5.40 \\
\hline$\rho(\tau, N X)=\rho(\Gamma, N X)$ & -0.06 & 0.36 \\
\hline$\rho(\tau, Y)=\rho(\Gamma, Y)$ & 0.55 & -0.10 \\
\hline
\end{tabular}

Les conditions d'optimalité permettent d'identifier la cause des échecs du modèle. En posant $\lambda_{i t} P_{i t}^{C}=\Lambda_{i t}$, l'utilité marginale de la richesse en termes de paniers de biens de consommation, la combinaison des équations (16) et (5) conduit à

$$
\Gamma_{t}=\frac{\Lambda_{2 t}}{\Lambda_{1 t}}=\frac{\frac{\partial U}{\partial C_{2 t}^{C}}}{\frac{\partial U}{\partial C_{1 t}^{C}}}=\frac{C_{1 t}^{C}}{C_{2 t}^{C}}
$$

L'équation (18) illustre la mutualisation des risques par les ménages. Ces derniers s'assurent en termes de paniers de biens de consommation. L'équation (18) établit une relation entre le 
rapport des consommations entre les deux pays et les mouvements du taux de change réel. Cette expression résulte de la complétude des marchés financiers et de la séparabilité des préférences.

Lorsque les préférences sont symétriques $(\gamma=0.5)$, la composition des paniers de consommation des deux ménages est identique. Le taux de change réel, interprétable comme le prix relatif des paniers de biens, est alors constant (equation (3)). En revanche, dès lors que $\gamma \neq 0.5$ (en particulier, lorsque $\gamma>0.5$, le panier de consommation est constitué de plus de bien local que de bien étranger, ce qui est le cas après étalonnage de notre modèle), la composition des paniers de consommation diffère, ce qui engendre les fluctuations du taux de change réel. L'écart à la PPA introduite par la préférence pour les biens nationaux engendre une volatilité du taux de change réel. Toutefois, elle s'avère bien inférieure à celle observée dans les pays du G7.

De plus, l'équation (18) montre que la faible volatilité du taux de change réel se traduit par une grande corrélation internationale entre les niveaux de consommation. Au terme des simulations, la corrélation croisée des consommations s'avère trop élevée par rapport aux données.

Nous étudions, dans la section suivante, la sensibilité de ces conclusions à deux paramètres essentiels du modèle : le taux d'importation et le degré de substituabilité entre les biens.

\subsection{Etude de sensibilité}

A la suite d'un choc technologique dans le pays 1, la corrélation croisée des productions s'explique par un effet volume et un effet prix :

- Effet volume : la demande en bien 2 en provenance des ménages du pays 1, pour motif de consommation, et de l'entreprise 1, pour motif d'investissement, augmente, ce qui favorise la production du pays 2. Cette dernière s'accroît d'autant plus que le taux d'importation $(1-\gamma)$ des agents du pays 1 est élevé.

- Effet prix : Cet effet volume, qui favorise une évolution symétrique des productions nationale et étrangère, est atténué par un effet prix : la baisse du prix du bien 1 relativement au prix du bien 2 incite tous les ménages et toutes les entreprises à demander plus de biens 1 au détriment du bien 2. L'ampleur de cet effet prix, qui induit une augmentation de la production du bien 1 au détriment de la production du bien 2, dépend de l'élasticité de substitution entre les biens $\theta$. Ce paramètre a de plus des effets sur l'ampleur de la réponse des termes de l'échange $\tau$ au choc technologique. Le graphique 4 illustre les mécanismes qui expliquent ce phénomène. Sur le graphique 4 sont reportés les différentiels d'offre et de demande sur les marchés des biens 1 et 2. L'expansion de la productivité globale dans le pays $1\left(a_{1}\right)$ se traduit par une hausse de $y_{1}^{s}$ soit un déplacement du différentiel d'offres de biens vers la gauche. La pente de la courbe de demande dépend de $\theta$ l'élasticité de substitution entre les biens. Lorsque les biens sont très substituables ( $\theta$ élevé), il suffit d'une faible variable des termes de l'échange $\tau$ pour redistribuer la demande en faveur d'un bien au détriment de l'autre. Lorsque $\theta$ est faible, l'équilibre s'établit en $E_{1}$, caractérisé par 
FIG. 4 -Effets d'une modification de l'élasticité de substitution entre les biens ( $\theta)$ sur les termes de l'échange et le différentiel de production

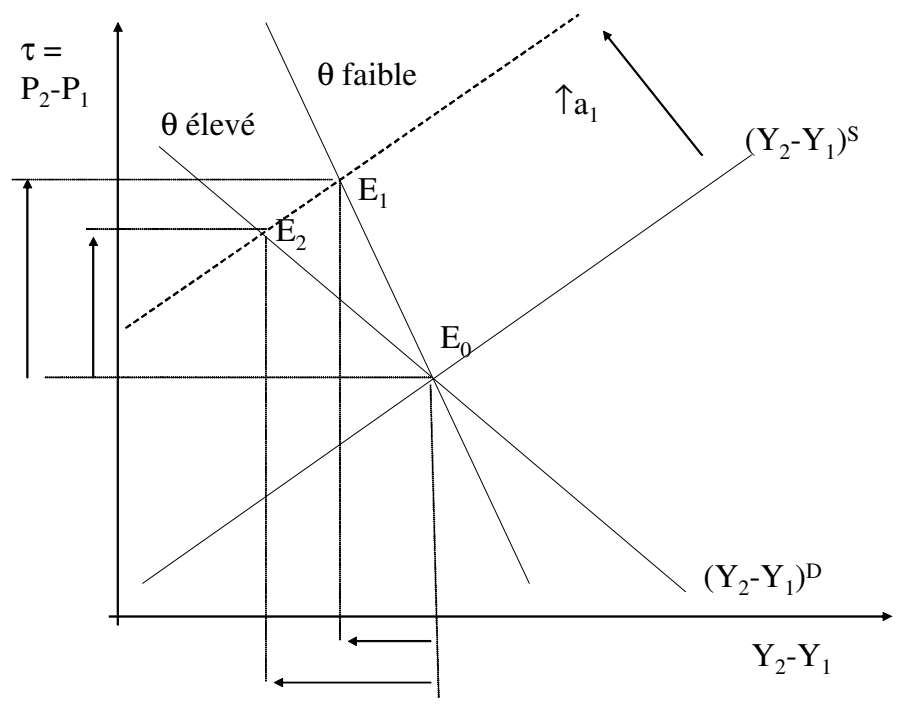

une réponse de $\tau$ de plus grande ampleur et un différentiel de production plus faible que lorsque $\theta$ est élevé (point $E_{2}$ sur le graphique 4).

Une plus forte complémentarité entre les biens tendrait à rendre plus positive la corrélation internationale des productions et à accroître la volatilité des termes de l'échange, donc celle du taux de change réel (équation (3)). Les simulations numériques (tableau 7) confirment cette intuition. Les corrélations internationales des productions, des heures travaillées et des niveaux d'investissement sont plus élevées pour un faible degré de substituabilité des biens. De même, les termes de l'échange ainsi que le taux de change réel voient leur écart-type s'accroître tandis que la corrélation croisée des consommations se rapproche des faits stylisés en devenant plus modérée. Toutefois, l'ampleur de ces effets n'est pas quantitativement suffisante pour rendre le modèle plus proche des faits stylisés. Dans les sections suivantes, les travaux proposant des modèles avec plusieurs biens tentent de reproduire la corrélation positive des productions en atténuant l'effet prix (ou effet termes de l'échange) qui engendre des évolutions asymétriques des productions.

Le modèle international canonique ne parvient pas à expliquer deux faits stylisés saillants. Les données font apparaître une synchronisation cyclique plus prononcée des productions que des consommations. En raison de la complétude des marchés financiers et de la séparabilité des préférences, cette hiérarchie des corrélations croisées est inversée dans le modèle. Cette première insuffisance du modèle constitue le "quantity puzzle" dans la terminologie de Backus et al. (1995). De plus, le modèle ne parvient pas à expliquer l'ampleur des fluctuations du taux de change réel dont la volatilité demeure insuffisante au regard des faits stylisés. Pour reprendre 
TAB. 7 - Impact de $\theta$ sur les corrélations croisées et la volatilité du taux de change réel

\begin{tabular}{|c|c|c|c|}
\hline & $\theta=1.5$ & $\theta=0.5$ & Faits stylisés (Médiane) \\
\hline$\rho\left(Y_{1}, Y_{2}\right)$ & 0.10 & 0.25 & 0.39 \\
\hline$\rho\left(C_{1}^{C}, C_{2}^{C}\right)$ & 0.79 & 0.63 & 0.35 \\
\hline$\rho\left(H_{1}, H_{2}\right)$ & 0.07 & 0.19 & 0.29 \\
\hline$\rho\left(I_{1}^{C}, I_{2}^{C}\right)$ & -0.13 & -0.06 & 0.36 \\
\hline$\frac{\sigma_{\tau}}{\sigma_{Y}}$ & 0.32 & 0.40 & 3.50 \\
\hline$\frac{\sigma_{\Gamma}}{\sigma_{Y}}$ & 0.18 & 0.23 & 5.40 \\
\hline$\rho(\tau, N X)$ & -0.06 & -0.40 & -0.10 \\
\hline$\rho(\tau, Y)$ & 0.50 & 0.52 & 0.36 \\
\hline
\end{tabular}

les termes de Backus et al. (1995), cet échec est dénommé le "price puzzle". Comme le montre l'analyse de sensibilité, ces énigmes empiriques constituent des questions connexes.

\section{Résoudre les énigmes empiriques}

L'abondante littérature suscitée par ces deux énigmes empiriques atteste de la vitalité de ce courant de recherche. Notre ambition n'est pas de fournir une liste exhaustive des travaux mais de souligner les intuitions qui expliquent les amendements au modèle canonique proposés dans la littérature.

Nous avons choisi de mettre l'accent sur deux dimensions. La première tente de résoudre le "quantity puzzle" en réduisant la corrélation croisée des consommations, cette dernière étant trop élevée par rapport aux faits stylisés. Cette approche tente d'atténuer les effets de la complétude des marchés financiers en introduisant des éléments non assurables dans la fonction d'utilité ou en considérant des marchés financiers incomplet. Notons que, si ces travaux s'attachent à rendre compte de la corrélation des consommations, les mécanismes étudiés ne sont pas sans conséquence sur l'interdépendance des heures travaillées, donc de la production et de l'investissement. En effet, rappelons en particulier que la complétude des marchés conduit à un partage égalitaire des effets richesse ce qui conduit à une évolution asymétrique des heures travaillées. En réduisant les effets de la complétude des marchés, les contributions présentées dans la section suivante sont susceptibles de modifier aussi les résultats du modèle concernant l'interdépendance des productions et des niveaux d'emploi et d'investissement.

Une seconde voie de recherche étudie le rôle des interdépendances productives. Les entreprises utilisent dans leur processus de production des biens produits par ses partenaires commerciaux. Ces échanges de biens intermédiaires seraient susceptibles d'accroître la corrélation internationale des productions jusqu'à un niveau conforme aux données. 


\subsection{L'interdépendance internationale des consommations}

La complétude des marchés financiers rend les corrélations internationales des consommations supérieures à celle des faits stylisés et incite, par un effet richesse positif, le ménage étranger à réduire son offre de travail, engendrant ainsi une pression à la baisse de la production étrangère. La mutualisation des risques associée à des préférences additivement séparables engendre une forte interdépendance des consommations et des évolutions asymétriques des heures travaillées.

Les contributions présentées ci-après tentent d'atténuer les effets de la complétude des marchés financiers afin de rendre les modèles mieux à même de reproduire l'interdépendance internationale des consommations. Nous classerons ces travaux en deux courants :

- Le premier explore les effets d'une non séparabilité des préférences

- Le second cherche à réduire l'ampleur des effets richesses qui affectent le pays étranger

\subsubsection{La non séparabilité des préférences}

La complétude des marchés financiers associée à une fonction d'utilité additivement séparable engendre une forte corrélation croisée des consommations, caractéristique contraire aux faits stylisés. En revanche, si la consommation intervient de manière non séparable avec un élément spécifique à chaque pays, l'égalisation de effets richesse entre les deux pays ne se traduit pas nécessairement par une corrélation parfaite des consommations.

Bec (1994) propose, à la manière de Christiano \& Eichenbaum (1992), d'introduire les dépenses publiques dans la fonction d'utilité.

$$
u\left(c_{i t}, g_{i t}, l_{i t}\right)=\log \left(c_{i t}+\alpha_{g} g_{i t}\right)+\gamma \log \left(1-n_{i t}\right)
$$

avec $\gamma>0$ et $\alpha_{g} \in[0,1] . c_{i t}$ désigne la quantité de bien consommée par le ménage du pays $i$, $g_{i t}$ la dépense publique par ménage $i$. Les dépenses publiques procurent à l'agent représentatif autant d'utilité que $\alpha_{g}$ unités de consommation privée. En posant $\alpha_{g}=1$, consommations privée et publique sont parfaitement substituables. En revanche, pour $\alpha_{g}=0$, seule la consommation individuelle est source de bien-être.

Bec (1994) retient l'hypothèse de marchés financiers complets, ce qui se traduit par l'égalisation des multiplicateurs associés aux contraintes budgétaires de chaque pays (equation (16)). A l'instar du modèle canonique, les multiplicateurs de Lagrange sont égaux à l'utilité marginale associée à la consommation (equation (4)). On obtient donc

$$
\frac{1}{c_{1 t}+\alpha_{g} g_{1 t}}=\frac{1}{c_{2 t}+\alpha_{g} g_{2 t}}
$$

Les chocs de dépenses publiques, spécifiques à chaque pays, seraient de nature à réduire la corrélation croisée des consommations. Une telle spécification, suggérée par Barro (1981), parviendrait à réduire la corrélation croisée des consommations à condition de poser $\alpha=1$ afin 
d'amplifier l'impact des dépenses publiques sur l'utilité marginale de la consommation. Or, si l'on en croit l'estimation d'Aschauer (1985), $\alpha$ avoisinerait 0,2. Pour une telle valeur de $\alpha$, la corrélation croisée des consommations demeure malheureusement proche de l'unité.

Par extension, on peut imaginer des chocs spécifiques qui interviennent directement de manière non séparable dans la fonction d'utilité tels que des chocs de préférence spécifiques à chaque pays.

Les biens qui font l'objet d'un commerce à l'échelle internationale sont dits échangeables tandis que ceux qui ne peuvent être utilisés que dans le pays où ils ont été produits (tels que les services, les transports, la construction) sont qualifiés de biens non échangeables. Dans le modèle de Stockman \& Tesar (1995), le ménage domestique consomme du loisir, des biens échangeables domestique et étranger ainsi que le bien non échangeable national. Les auteurs imposent une non séparabilité des biens échangeables/non échangeables dans la fonction d'utilité et introduisent des chocs de préférence spécifiques à chaque pays, lesquels viennent effectivement réduire la corrélation internationale des consommations.

Le loisir notamment constitue un bien "non échangeable". Afin de réduire la corrélation des consommations, il convient de renforcer l'effet du loisir dans les choix de consommation. Cela est possible si le loisir intervient de manière non séparable avec la consommation. Devereux et al. (1992) montrent que, dans ce cadre, le modèle parvient à engendrer une corrélation internationale des consommations conformes aux données.

\subsubsection{Réduire le partage international des risques}

Eliminer l'effet richesse : L'effet richesse positif dont bénéficie le ménage qui ne subit pas le choc favorable est à l'origine de l'asymétrie des réponses des heures travaillées. Hairault (1998) propose donc d'accentuer la covariation des heures travaillées en considérant une fonction d'utilité qui élimine les effets richesse dans les décisions d'offre de travail des ménages. A cette spécification des préférences à la Greenwood et al. (1988) s'ajoute un effet lié aux termes de l'échange dans les choix des agents ${ }^{4}$. Hairault (1998) développe un modèle à deux pays et deux biens similaire au modèle canonique à l'exception des préférences. La fonction d'utilité instantanée du ménage $i$ devient

$$
U\left(C_{i t}^{C}, 1-H_{i t}\right)=\ln \left(C_{i t}^{C}+G\left(1-H_{i t}\right)\right) \quad i=1,2
$$

où $G\left(1-H_{i t}\right)=\gamma \frac{\left(1-H_{i t}\right)^{1-\sigma}}{1-\sigma}$. La condition du premier ordre relative à la consommation est

$$
\frac{1}{C_{i t}^{C}+G\left(1-H_{i t}\right)}=\lambda_{i t} P_{i t}^{C} \quad i=1,2
$$

\footnotetext{
${ }^{4}$ La spécification de Greenwood et al. (1988) est utilisée par Devereux et al. (1992). Toutefois, ces derniers n'introduisent pas d'effets liés à la variabilité des termes de l'échange.
} 
Sachant (19), le choix de loisir est, dans chaque pays,

$$
\begin{aligned}
G^{\prime}\left(1-H_{1 t}\right) & =\frac{w_{1 t}}{P_{1 t}^{C}} \\
G^{\prime}\left(1-H_{2 t}\right) & =\tau_{t} \frac{w_{2 t}}{P_{2 t}^{C}}
\end{aligned}
$$

(20) et (21) ne font pas intervenir les utilités marginales de la richesse $\lambda_{i t}$. Les choix relatifs à l'offre de travail des ménages relèvent d'une décision intratemporelle. A la suite d'un choc de productivité dans le pays 1, les termes de l'échange se détériorent, ce qui accroît la rémunération des heures travaillées dans le pays étranger (équation (21)). Le ménage souhaite donc travailler davantage. La hausse de l'emploi d'équilibre qui en résulte favorise la productivité du capital et donc l'investissement. La conjugaison de l'effet des termes de l'échange et de l'absence d'effet richesse dans le choix de loisir produit donc un mouvement symétrique des heures travaillées, donc des productions et des niveaux d'investissement. En outre, par l'équation (19), la non séparabilité entre consommation et loisir amoindrit la corrélation croisée des consommations. Hairault (1998) parvient ainsi à reproduire l'interdépendance des productions et des consommations.

Marchés incomplets : La séparabilité de la fonction d'utilité conjuguée à la complétude des marchés financiers conduit à une corrélation parfaite des consommations. Les travaux évoqués précédemment lèvent l'hypothèse afférente aux préférences. Baxter \& Crucini (1995) ainsi que Kollman (1996) s'attachent à l'analyse de modèles qui n'autorisent pas un partage international des risques spécifiques. En particulier, lorsque les agents ont uniquement accès à des titres certains, ils sont dans l'impossibilité de diversifier les risques nationaux.

Baxter \& Crucini (1995) développent un modèle international à un bien dans lequel les titres à la disposition des ménages sont désormais des titres certains qui donnent lieu au paiement d'une unité de bien demain quel que soit l'état de la nature. Le ménage du pays $i$ reçoit en début de période le paiement attaché à la possession de $B_{i t}$ titres certains et procède à l'achat de $B_{i t+1}$ titres certains au prix $R_{t}$. La contrainte budgétaire du ménage du pays $i$ devient

$$
C_{i t}+R_{t} B_{i t+1} \preceq B_{i t}+w_{i t} H_{i t}
$$

Le ménage $i$ maximise son utilité intertemporelle sous (22), contrainte à laquelle est associée le multiplicateur $\lambda_{i t}$. La condition du premier ordre associée au choix de $B_{i t+1}$ conduit à

$$
R_{t} \lambda_{i t}=\beta E_{t}\left[\lambda_{i t+1}\right] \quad \forall i=1,2
$$

soit, dans les deux pays,

$$
R_{t}=\beta \frac{E_{t}\left[\lambda_{1 t+1}\right]}{\lambda_{1 t}}=\beta E_{t} \frac{E_{t}\left[\lambda_{2 t+1}\right]}{\lambda_{2 t}}
$$

Dans le modèle log-linéarisé, cette expression devient

$$
E_{t}\left[\hat{\lambda}_{1 t+1}\right]-E_{t}\left[\hat{\lambda}_{2 t+1}\right]=\hat{\lambda}_{1 t}-\hat{\lambda}_{2 t}
$$


FIG. 5 - Réponses à un choc technologique asymétrique dans le pays 1 lorsque les marchés financiers sont incomplets (modèle à deux pays et un bien)
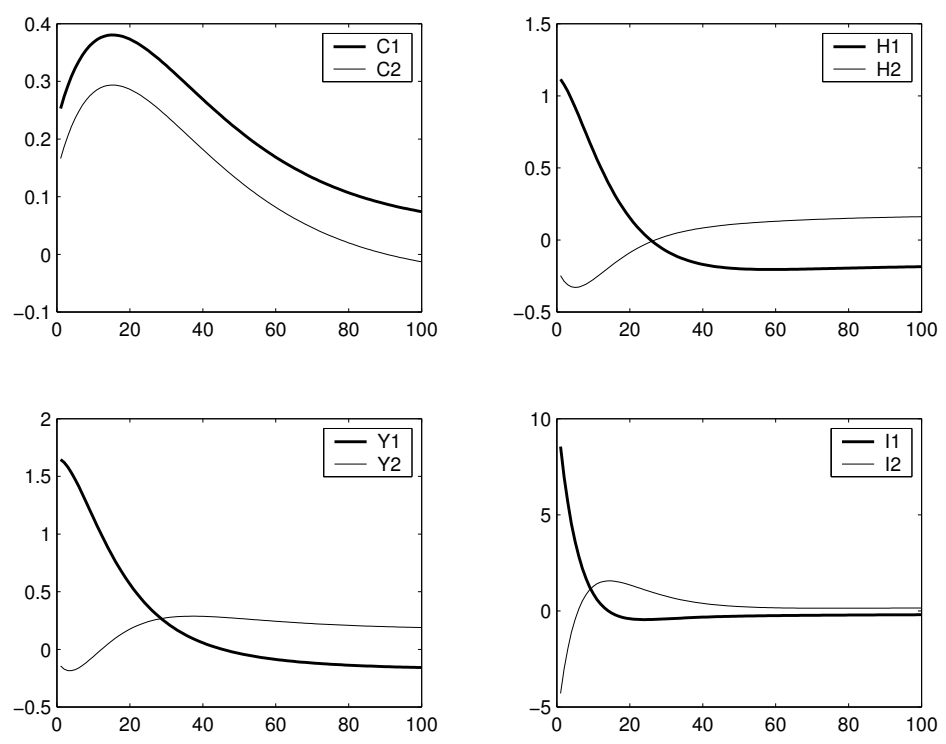

Le différentiel des multiplicateurs de Lagrange, interprétables comme la répartition de la richesse entre les deux pays, suit une martingale. L'équation (23) introduit des effets richesse permanents dans les comportements intertemporels. Or, le ratio des multiplicateurs $\lambda$ dans les 2 pays sont liés au rapport des utilités marginales associées à la consommation dans les 2 pays (équation (18)). Les transferts de richesse permanents, qui se traduisent par des réponses différentiées de $\lambda$ dans les deux pays, devraient donc réduire la corrélation croisée des consommations.

Pour illustrer ce point, nous reconstruisons le modèle de Baxter \& Crucini (1995) et reportons sur le graphique 5 les fonctions de réponse obtenues après un choc technologique asymétrique favorable dans le seul pays 1 .

Les variables convergent vers un nouvel état stationnaire. L'effet richesse positif dont bénéficie le ménage 1 lui permet à long terme d'atteindre un niveau de consommation plus élevé et de réduire durablement ses heures travaillées. On observe, à long terme, des évolutions inverses pour le ménage étranger : sur le graphique 5, les heures travaillées dans le pays 2 sont à long terme plus élevées qu'à l'état stationnaire initial, tandis que la consommation s'établit à un niveau inférieur à celui qui prévalait à l'état stationnaire initial. Toutefois, quantitativement, si l'incomplétude des marchés rapproche la corrélation internationale des consommations des faits stylisés, Baxter \& Crucini (1995) et Kollman (1996) montrent que cet effet n'est pas assez puissant pour renverser l'ordre des corrélations croisées des consommations et des productions. 5

\footnotetext{
${ }^{5}$ L'incomplétude des marchés financiers rend le modèle non stationnaire, ce qui viendrait invalider la méthode de résolution fondée sur une linéarisation autour de l'état stationnaire prévalant avant le choc. Afin de rendre
} 
Kehoe \& Perri (2002) proposent une représentation alternative de l'incomplétude des marchés financiers. Chaque pays a la possibilité de manquer à sa promesse de rembourser ses dettes. Dans ce cas, le débiteur est exclu des marchés financiers et se retrouve en situation d'autarcie. Un pays $i$ subit une contrainte d'incitation à rembourser telle que l'utilité intertemporelle de son ménage s'il rembourse sa dette est supérieure à celle qu'il aurait s'il était exclu des marchés financiers. L'introduction de ce type de contrainte permet de rapprocher les résultats du modèle des faits stylisés. En particulier, le modèle parvient presque à expliquer le "quantity puzzle". La corrélation croisée des consommations (0.29) est de l'ordre de celle obtenue pour les productions (0.25). En outre, la corrélation croisée des heures travaillées et de l'investissement est positive, caractéristique que le modèle canonique ne parvient pas à reproduire.

Kehoe \& Perri (2002) expliquent les raisons de ce succès de la manière suivante. Supposons qu'il existe un planificateur central mondial qui tente de réaliser les allocations correspondant aux marchés complets. Le pays dans lequel se produit le saut de la productivité globale (le pays 1 par exemple) est peu désireux de partager les fruits de cette conjoncture favorable, ce qui rend attractif la situation d'autarcie. De plus, si les capitaux sont libres d'affluer dans ce pays, l'autarcie constitue une option encore plus intéressante. Afin d'inciter le pays à respecter ses engagements vis-à-vis de ses créanciers, le planificateur doit restreindre les entrées de capitaux, ce qui se traduit par une corrélation croisée positive de l'investissement. Cette interdépendance de l'investissement implique une corrélation positive des heures travaillées. En effet, l'accroissement du stock de capital augmente la productivité du travail, ce qui incite les ménages des deux pays à augmenter leur offre de travail.

En outre, la complétude des marchés financiers obligerait le ménage qui jouit d'un accroissement de sa richesse à le partager avec son homologue étranger, ce qui l'inciterait à renoncer à participer aux marchés financiers internationaux. Afin de persuader le ménage d'écarter cette option, le planificateur doit autoriser une hausse significative et persistante de la consommation nationale par rapport à la consommation étrangère. Ce mécanisme explique la corrélation modérée entre les consommations des deux pays.

\subsection{L'interdépendance internationale des productions}

Le modèle canonique conduit à une interdépendance plus prononcée des consommations que des productions, alors que l'ordre des corrélations est inversé dans les données. Afin d'expliquer le "quantity puzzle", les travaux évoqués ci-dessus tentent de réduire l'interdépendance des consommations. En revanche, les contributions présentées dans cette section recherchent les mécanismes susceptibles d'augmenter la corrélation croisée des productions.

le modèle stationnaire, il est possible d'incorporer un facteur d'escompte endogène ou une prime de risque sur le taux d'intérêt national proportionnelle à la dette extérieur. Schmitt-Grohe \& Uribe (2003) démontrent que les modèles à marchés incomplets rendus stationnaires par ces différentes méthodes produisent des résultats quantitatifs similaires à ceux de Baxter \& Crucini (1995) et Kollman (1996). 


\subsubsection{Multiplicité des pays}

Zimmermann (1997) souligne qu'un modèle à trois pays serait susceptible de rendre compte des corrélations internationales des productions. A la suite d'un choc technologique asymétrique, la fuite des capitaux est subie par les deux autres pays. On devrait donc observer sur ces derniers une covariation positive de l'investissement, donc de l'emploi et des productions. Zimmermann (1997) applique cette approche à 3 pays : un petit pays, un pays voisin de grande taille et le reste du monde. Le modèle est étalonné pour le Canada/Les Etats-Unis/le reste du monde ainsi que la Suisse/l'Europe/le reste du monde.

Zimmermann (1997) parvient à reproduire la forte corrélation des produits observée entre pays voisins. L'interdépendance prononcée des productions est reproduite pour les couples Canada/Etats-Unis et Suisse/Europe. Toutefois, ce mécanisme tient plus à la dynamique jointe des résidus de Solow qu'aux mécanismes de propagation inhérents au modèle. En effet, l'estimation du processus des résidus de Solow fait apparaître une transmission internationale instantanée plus accentuée entre pays voisins. En outre, la corrélation internationale des productions demeure inférieure à celle des consommations. Le modèle s'écarte en cela des faits stylisés.

\subsubsection{Multiplicité des secteurs/multiplicité des biens}

Afin d'expliquer l'interdépendance des productions, des travaux étudient l'importance quantitative de deux mécanismes. Le premier a trait à la structure multi-sectorielle des économies tandis que la seconde met l'accent sur les échanges commerciaux liés à la présence de biens intermédiaires dans le processus de production.

Modèles multi-sectoriels La présence de plusieurs secteurs dans l'économie pourrait atténuer les mouvements asymétriques des capitaux qui engendrent des corrélations négatives de l'investissement donc des heures travaillées et de la production. En effet, à la suite d'un choc technologique sectoriel asymétrique, la réallocation intersectorielle des capitaux pourrait atténuer l'ampleur de l'afflux de capitaux en provenance de l'étranger. La structure multi-sectorielle réduit l'afflux des capitaux étrangers à l'origine de la corrélation négative de l'investissement puisque du capital peut être transféré depuis un autre secteur de l'économie. Cette intuition est mise en oeuvre par Stockman \& Tesar (1995) dans le cadre d'un modèle distinguant un secteur de biens échangeables et non échangeables dans chaque pays. Les auteurs aboutissent effectivement à une corrélation croisée des productions proches de celles observées dans les données. Toutefois, si le mécanisme permet de rendre compte de l'interdépendance des productions, il ne résout en rien la trop forte corrélation croisée des consommations.

Le modèle de Long \& Plosser (1983) développé en économie fermée incorpore une transmission intersectorielle positive des fluctuations. Le mécanisme est simple : l'activité de chaque secteur utilise, dans son processus de production, des biens intermédiaires produits par les autres 
secteurs. Un choc technologique sectoriel relance l'activité dans un secteur, ce qui se traduit par une expansion de la demande de biens en provenance des autres secteurs de l'économie. Le degré de substituabilité des biens dans la fonction de production règle l'ampleur de ce phénomène. L'extensions des mécanismes de Long \& Plosser (1983) à un contexte d'économie internationale pourrait expliquer l'interdépendance des productions entre les principaux pays industrialisés. Costello \& Praschnik (1993) évaluent la pertinence empirique de cette intuition, dans le cadre d'un modèle à deux pays et un secteur de bien intermédiaire. Ils obtiennent une corrélation internationale des productions plus élevée que celle de Backus et al. (1995) mais négligent de reporter les corrélations croisées des niveaux d'emploi et d'investissement.

Biens intermédiaires Ambler et al. (2002) proposent une synthèse des deux approches en introduisant une structure multisectorielle dont les processus de production nécessitent l'usage de biens intermédiaires. Les interdépendances productives conjuguées à la présence de plusieurs secteurs conduisent à une corrélation internationale des productions supérieure à celle obtenue par Backus et al. (1995). Toutefois, l'ordre des corrélations de la consommation et de la production demeure contraire aux données. La présence de marchés complets associée à des préférences additivement séparables rendent unitaire la corrélation croisée des consommations.

Alors que les contributions précédentes sont développées dans un cadre walrasien, Huang \& Liu (2006) incorporent avec succès l'approche de Ambler et al. (2002) à un cadre intégrant une concurrence monopolistique associée à des prix rigides. Huang \& Liu (2006) montrent que la combinaison d'une structure productive avec biens intermédiaires et de la rigidité nominale rend le modèle capable de reproduire la hiérarchie et le signe des corrélations internationales de la production, la consommation, l'emploi et l'investissement.

La production du bien final dans chaque pays s'effectue selon une chaîne de production à $N$ étapes (par exemple, la production d'une voiture nécessite un assemblage successif de biens intermédiaires). La production du bien intermédiaire à l'étape $n$ de la chaîne de production nécessite l'utilisation des biens intermédiaires des $n-1$ étapes précédentes, ces biens étant produits nationalement et importés. Par ailleurs, les producteurs de biens intermédiaires en concurrence monopolistique font face à des rigidités nominales : à chaque date, seule la moitié d'entre eux ont la possibilité de modifier leur prix.

A la suite d'un choc monétaire favorable dans le pays national, en raison de la chaîne de production, l'ajustement des prix et donc celui de la production est progressif. En effet, seule la moitié des producteurs situés au bas de la chaîne de production (les producteurs de matières premières, étape 1 de la production) répercutent sur leur prix la hausse du coût marginal des facteurs de production (capital et travail). Cette hausse du prix des matières premières constitue un renchérissement du coût de production pour des producteurs à l'étape suivante de la chaîne de production. Toutefois, cette hausse du coût marginal à l'étape 2 de la production est atténuée 
par le fait que seule la moitié des producteurs de matières premières (étape 1) ont procédé à un relèvement de prix. Par ailleurs, à l'étape 2 , à nouveau, seule la moitié des producteurs ont la possibilité d'augmenter leur prix. Ainsi, à l'étape 3 de la chaîne de production, les producteurs subissent une hausse bien moindre de leur coût marginal, etc ... Accroître le nombre d'étapes dans la chaîne de production revient à accentuer l'ampleur de la rigidité de prix. L'augmentation de la production du bien final dans le pays national s'avère donc graduel.

Dans le pays étranger, la hausse de la production nationale a pour conséquence un accroissement de la demande pour les biens intermédiaires étrangers à toutes les étapes de la production. La hausse de la production étrangère vient accroître la demande pour les facteurs de production étrangers. Leur prix va donc augmenter. L'économie étrangère subit donc la même incitation à augmenter ses prix à toutes les étapes de production, avec le même ajustement progressif que celui observé dans le pays national. Le modèle prédit alors une hausse de la production étrangère.

Huang \& Liu (2006) montrent que 4 étapes de production suffisent pour rendre compte de la corrélation croisée des productions et des consommations. Les raisons du succès du modèle concernant la corrélation internationale des productions sont simples.

- Effet volume : Un choc monétaire positif se traduit par une hausse de la consommation et de l'investissement dans le pays qui jouit de cette politique monétaire favorable. Or, la consommation et l'investissement se composent de biens nationaux et étrangers. La demande pour les biens produits dans les deux pays augmente, ce tend à rendre le modèle conforme à la corrélation internationale positive des productions. Cet effet volume est toutefois atténué par un effet prix.

- Effet prix : La dépréciation de la monnaie nationale, à la suite du choc monétaire, dégrade les termes de l'échange : les biens nationaux sont relativement moins chers, ce qui favorise la production de l'un des pays au détriment de l'autre. Ce mécanisme tend à engendrer une corrélation internationale négative des productions.

La présence de la chaîne de production associée à des prix rigides diminue l'ampleur de l'ajustement des prix et vient donc atténuer les variations des termes de l'échange. L'effet volume l'emporte sur l'effet prix.

\subsection{Dynamique du taux de change réel et rigidités nominales}

\subsubsection{La Nouvelle Macroéconomie Internationale}

La forte corrélation entre les dynamiques de court terme des taux de change réel et nominaux, mise en évidence par Mussa (1986) et confirmée sur données récentes par le tableau 3, suggère la présence de rigidités de prix. La Nouvelle Macroéconomie Internationale (NMI) qui privilégie un cadre de concurrence monopolistique associée à une imparfaite flexibilité des prix pourrait donc nous permettre de mieux comprendre l'ampleur des fluctuations du taux de change réel. Obstfeld \& Rogoff (1995) jettent les bases du modèle qui constitue le paradigme de la NMI. L'économie 
compte deux pays, chacun produisant un continuum de biens différenciés. Les entreprises sont en situation de concurrence monopolistique. Obstfeld \& Rogoff (1995) retiennent l'hypothèse de l'absence de barrières aux échanges commerciaux de sorte que la loi du prix unique est vérifiée pour tous les biens. Les préférence des agents étant symétriques, le taux de change réel est alors constant. Aussi le modèle d'Obstfeld \& Rogoff (1995) est-il incapable d'expliquer les fluctuations du taux de change réel. Afin de pallier cette insuffisance, Betts \& Devereux (1996) proposent d'intégrer dans le cadre proposé par Obstfeld \& Rogoff (1995) la possibilité que la loi du prix unique ne soit pas vérifiée pour certains biens dans l'économie. En conséquence, en l'absence de la parité des pouvoirs d'achat, le taux de change réel n'est plus constant. Betts \& Devereux (1996) ouvre une voie de recherche qui attribue l'absence de la loi du prix unique au "Pricing-To-Market" ou "Local Currency Pricing".

La loi du prix unique repose sur l'arbitrage effectué par les consommateurs. En l'absence de coûts de transports ou de restrictions aux flux commerciaux, l'échange d'un bien conduit à l'égalisation de son prix en monnaie locale à celui exprimé en monnaie étrangère. Or, la présence de barrières commerciales et de coûts de transports, peut rendre cet arbitrage impossible. Les entreprises ont donc la possibilité de choisir un prix différent en fonction de la localisation de leur clientèle. Elles déterminent un prix en monnaie étrangère pour le marché étranger et un prix en monnaie locale pour le marché local. Ces deux prix, convertis dans la même monnaie, ne sont pas égaux. La loi du prix unique n'est pas vérifiée pour ce type de biens. Le "PricingTo-Market" ou "Local Currency Pricing" fait référence à cette discrimination de prix pratiquée par les entreprises exportatrices.

Deux types de littérature empirique confirment la pertinence empirique de ce comportement de choix de prix ${ }^{6}$. Le premier souligne l'importance de la discrimination de prix fondée sur la localisation de la clientèle. Engel \& Rogers (1996) comparent les mouvements des prix entre différent pays et ceux observés l'intérieur d'un pays. Ils observent que les différences de prix entre des villes américaines et canadiennes sont systématiquement plus variables que ces différences entre des villes aussi éloignées d'un même pays. Engel \& Rogers (1996) interprètent ces résultats comme une preuve que la discrimination de prix à l'échelle internationale est plus accentuée que celle pratiquée à l'échelle nationale. La frontière, plus que la distance, importe dans les comportements de discrimination de prix. Engel \& Rogers (2001) montrent la robustesse de cette conclusion sur données européennes.

Les entreprises qui pratiquent la discrimination de prix fixent ceux-ci dans la monnaie de l'acheteur. Les entreprises peuvent alors décider de ne pas répercuter l'intégralité des mouvements du taux de change nominal sur leurs prix, surtout s'il s'agit d'une appréciation de leur monnaie qui appellerait une hausse des prix destinés aux marchés étrangers. Cette stratégie

\footnotetext{
${ }^{6}$ Voir Goldberg \& Knetter (1997) pour une revue de cette littérature. Dans la suite de cet article, "discrimination de prix", "facturation dans la monnaie de l'acheteur" et "segmentation des marchés" seront synonymes.
} 
serait au coeur d'une pratique commerciale destinée à préserver leurs parts de marchés. Par exemple, au milieu des années 80, les constructeurs automobiles allemands n'ont pas baissé leurs prix de vente aux Etats-Unis à la suite de la forte appréciation du dollar américain. L'absence de sensibilité des prix aux modifications du taux de change nominal constitue une seconde illustration de la présence de discrimination de prix. Knetter (1993) confirme la pertinence empirique de ce phénomène en estimant l'élasticité des prix des exportations aux mouvements du taux de change nominal. Ces estimations sont effectuées sur des données annuelles désagrégées entre 1973 et 1987 pour le Royaume-Uni, les Etats-Unis, le Japon et l'Allemagne et montrent que le coefficient associé au fluctuations du taux de change nominal n'est pas unitaire : les prix à l'exportations sont partiellement insensibles aux mouvements du taux de change. De même, Feenstra \& Kendall (1997) observent qu'une part significative de la déviation à la loi du prix unique provient de la faible sensibilité des prix à l'exportation et des prix à l'importation aux mouvements du taux de change nominal, ce qui attesterait de la présence de discrimination de prix dans les relations entre les Etats-Unis et le Canada, l'Allemagne et le Royaume-Uni dans la période 1974-1994.

Un modèle intégrant la discrimination de prix pourrait non seulement expliquer les fluctuations du taux de change réel mais aussi rendre compte de l'interdépendance des productions, phénomène mal reproduit par les modèles de la littérature. En effet, lorsque les biens sont facturés dans la monnaie du vendeur, à la suite d'une dépréciation par exemple de la monnaie nationale, les biens nationaux sont relativement moins chers que les biens étrangers : par un effet prix, la production des biens nationaux s'accroît au détriment des productions étrangères. La redistribution de la demande en faveur des biens d'un seul pays engendre une corrélation négative des activités productives, phénomène que l'on n'observe pas dans les faits stylisés.

En revanche, lorsque les biens sont facturés dans la monnaie de l'acheteur, à la suite d'une dépréciation de la monnaie nationale, les entreprises qui pratiquent une discrimination de prix en fonction de la localisation de leurs clientèles peuvent décider de ne pas modifier leurs tarifs et de renoncer à une partie de leurs marges afin de préserver leurs parts de marchés. Les prix relatifs qui déterminent l'allocation des demande de biens ne sont donc pas sensibles aux variations du taux de change nominal. Pour les productions des entreprises qui pratiquent la discrimination de prix, nous n'observerons pas de redistribution des demandes de biens engendrant des mouvements asymétriques des productions. L'effet prix est limité en présence de "Local Currency Pricing" associée à des rigidités nominales. L'effet volume qui engendre une corrélation positive des productions rend alors le modèle conforme aux données.

Enfin, en présence de discrimination de prix, la loi du prix unique n'est pas vérifiée, ce qui amplifie les mouvements du taux de change réel. La discriminations de prix pourrait constituer une caractéristique intéressante pour rendre compte de la volatilité du taux de change réel.

Chari et al. (2002) montrent qu'un tel modèle parvient à rendre compte de la volatilité du 
taux de change réel. Les corrélations internationales des productions, consommations, emplois et investissements sont également bien reproduites. Cette conformité du modèle aux données est obtenue sous les hypothèses que les entreprises modifient en moyenne leur prix une fois l'an et que les ménages sont caractérisés par une forte aversion au risque.

\subsubsection{Un modèle de référence}

Le modèle international avec concurrence monopolistique et rigidités nominales développé par Obstfeld \& Rogoff (1995) s'est imposé comme un cadre théorique de référence en macroéconomie internationale. Smets \& Wouters (2005) y voient un outil essentiel pour la conduite de la politique monétaire. Les travaux empiriques confirment la pertinence de ce choix. Bergin (2002, 2006) estime un modèle international intégrant une concurrence monopolistique et des rigidités nominales. L'estimation par maximum de vraisemblance sur les pays du G7 confirme que le modèle avec rigidités nominales rend compte de la dynamique conjointe du produit, de la consommation, du taux de change et du compte courant. La discrimination de prix à la Betts \& Devereux (1996) est également validée par les données. Kollmann (2001) confirme que la présence du "Local Currency Pricing" rend le modèle capable de reproduire une forte volatilité du taux de change réel.

Toutefois, Obstfeld \& Rogoff (2000a) expriment des réserves quant à la pertinence empirique de la discrimination de prix. Leurs arguments reposent notamment sur deux points. Le premier reprend les conclusions du ECU Institute rappelant que dans les pays du G7 une grande part des exportations sont libellées en monnaie nationale, et non dans la monnaie de destination des exportations. Ce pourcentage s'élève à $92 \%$ aux Etats-Unis, 40\% au Japon, 77\% en Allemagne, $55 \%$ en France, $62 \%$ au Royaume-Uni, $40 \%$ en Italie et 43 aux Pays-Bas. De plus, la discrimination de prix implique un ajustement des taux de marge à la suite des mouvements du taux de change nominal. Gagnon \& Knetter (1995) observent un tel comportement dans le secteur automobile japonais tandis que le faible ajustement des taux de marge des constructeurs allemands et nord-américains semble indiquer l'absence de discrimination de prix. Le modèle de NMI de référence reprend donc la formulation d'Obstfeld \& Rogoff (1995), sans discrimination de prix.

\section{Une illustration des développements récents : Coûts de trans- port et biens échangés / non échangés}

Cette section est consacrée aux contributions récentes de la macroéconomie internationale. Elles présentent la particularité de renouveler le champ de recherche en liant la macroéconomie ouverte aux théories du commerce international et aux théories financières. Nous ne serons pas en mesure de présenter l'ensemble des développements récents. Nous choisissons de mettre l'accent sur l'ouverture vers les théories du commerce international. Ces dernières fournissent 
des éléments de réponse concernant la décision d'exporter des entreprises ainsi que leurs choix de localisation. Dans ces théories, la distinction entre biens échangés et non échangés devient endogène selon la décision des entreprises d'exporter ou non leur production. Dans cette décision, le coût de transport du bien constitue une variable essentielle. Ce mécanisme s'avère tout à fait pertinent dans l'analyse des fluctuations macroéconomiques à l'échelle internationale.

\subsection{Le rôle des biens non échangés dans l'explication des fluctuations du taux de change est tout d'abord minimisé}

Les modèles d'équilibre général dynamiques et stochastiques développés en économie ouverte ont, dans un premier temps, négligé la présence des coûts de transport dans les échanges commerciaux. Les biens dont les coûts de transport sont les plus élevés ne sont pas échangés. Certes, Stockman \& Tesar (1995) présentent un modèle international comprenant des secteurs de biens échangeables et non échangeables. Toutefois, Chari et al. (2002) et Engel $(1993,1999)$ soulignent qu'une telle structure multi-sectorielle ne présente aucun intérêt pour étudier les fluctuations du taux de change réel. Ils fondent leur conclusion sur la décomposition suivante des fluctuations du taux de change réel.

Soit $p_{t}$ le log de l'indice de prix à la consommation dans le pays national. Cet indice de prix est une moyenne pondérée des prix des biens échangeables $p^{T}$ et non échangeables $p^{N}$

$$
p_{t}=(1-\xi) p_{t}^{T}+\xi p_{t}^{N}
$$

$\xi$ désignant le poids des biens non échangeables dans l'indice de prix à la consommation. Ce dernier s'exprime de manière symétrique dans le pays étranger

$$
p_{t}^{*}=\left(1-\xi^{*}\right) p_{t}^{T *}+\xi^{*} p_{t}^{N *}
$$

Le taux de change réel en $\log \Gamma_{t}$ est défini comme

$$
\Gamma_{t} \equiv e_{t}+p_{t}^{*}-p_{t}
$$

avec $e_{t}$ le log du taux de change nominal. En utilisant (24) et (25), (26) devient

$$
\Gamma_{t}=\underbrace{e_{t}+p_{t}^{T *}-p_{t}^{T}}_{\text {prix relatif des biens échangeables }\left(\Gamma_{t}^{T}\right)}+\underbrace{\xi^{*}\left(p_{t}^{N *}-p_{t}^{T *}\right)-\xi\left(p_{t}^{N}-p_{t}^{T}\right)}_{\text {prix relatif des biens échangeables et non échangeables }\left(\Gamma_{t}^{N}\right)}
$$

L'équation (27) montre que le taux de change réel se décompose en deux éléments : 1) le prix relatif des biens échangeables (ou déviation à la loi du prix unique) noté $\Gamma_{t}^{T}$ et 2) le prix relatif des biens échangeables et non échangeables noté $\Gamma_{t}^{N}$. Chari et al. (2002) et Engel $(1993,1999)$ montrent que le prix relatif des biens échangeables et non échangeables joue un rôle négligeable dans l'explication de la forte volatilité du taux de change réel. Le graphique 6 illustre ce point. 
Fig. 6 - Décomposition du taux de change réel $\Gamma$ en ses deux éléments : prix relatif des biens échangeables $\Gamma^{T}$ et prix relatif des biens échangeables et non échangeables $\Gamma^{N}$. (Source : Chari et al. (2002), indices de prix à la consommation trimestriels, taux de change entre les Etats-Unis et un agrégat de pays européens.)

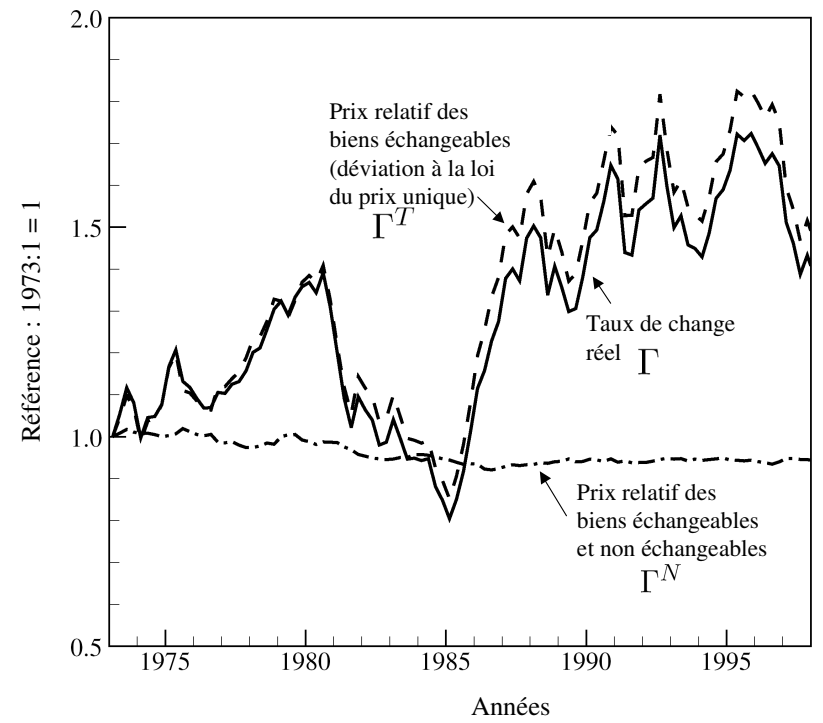

Les fluctuations du taux de change réel $\Gamma$ semblent essentiellement être expliquée par la déviation à la loi du prix unique $\Gamma^{T}$. L'analyse du prix des biens non échangeables semblent donc inutile dans la compréhension des fluctuations du taux de change réel. Cette position est aujourd'hui remise en cause par des travaux théoriques et empiriques.

Sur le plan théorique, Hairault \& Sopraseuth (2005) et Hau (2000) montrent que la faible volatilité du prix relatif des biens échangeables et non échangeables ne signifie pas qu'il ne joue aucun rôle dans les fluctuations du taux de change réel. D'après Hairault \& Sopraseuth (2005), le prix relatif des biens échangeables et non échangeables $\Gamma^{N}$ est certes moins volatile que la déviation à la loi du prix unique $\Gamma^{T}$ mais les deux éléments du taux de change réel sont significativement négativement corrélés. Les variations du prix relatif des biens échangeables et non échangeables viennent donc atténuer l'ampleur des fluctuations du taux de change réel. Dans le modèle de Chari et al. (2002) à une structure multisectorielle (biens échangeables / non échangeables), Hairault \& Sopraseuth (2005) soulignent que les chocs monétaires engendrent des réallocations sectorielles entre les entreprises produisant des biens échangés et celles produisant des biens non échangés, ce qui vient affecter la dynamique des prix relatifs des biens échangeables et non échangeables et donc celle du taux de change réel.

Obstfeld \& Rogoff (2000b) plaident en faveur de la prise en compte des coûts de transport dans les modèles d'économie ouverte car ces derniers seraient en mesure de résoudre les énigmes empiriques de la macroéconomie internationale. L'accent sur les coûts de transport ouvre la voie 
vers une réflexion plus large sur la distinction entre biens échangés et biens non échangés. Nous présentons les principales équations de Obstfeld \& Rogoff (2000b) afin de rendre transparente l'intuition des résultats.

Considérons un modèle à deux pays et deux biens. Dans le pays national, la consommation est fonction des biens national $\left(C_{H}\right)$ et étranger $\left(C_{F}\right)$

$$
C=\left(C_{H}^{\frac{\theta-1}{\theta}}+C_{F}^{\frac{\theta-1}{\theta}}\right)^{\frac{\theta}{\theta-1}} \quad 0<\theta<1
$$

$C_{H}^{*}$ et $C_{F}^{*}$ désignent les consommations par le ménage étranger de bien national et étranger. Les productions de biens national et étranger sont exogènes. Soit $P_{H}\left(P_{F}\right)$ le prix national du bien national (du bien étranger) et $P_{H}^{*}\left(P_{F}^{*}\right)$ le prix étranger du bien national (du bien étranger). Tous les prix sont exprimés en termes d'une monnaie commune. Les coûts de transport $\tau$ sont de type "iceberg" : pour chaque unité de bien national (bien étranger) exportée, seule une fraction $1-\tau$ arrive à l'étranger (dans le pays national). Si les marchés sont compétitifs, l'arbitrage implique

$$
\begin{aligned}
P_{F} & =\frac{P_{F}^{*}}{1-\tau} \\
P_{H} & =(1-\tau) P_{H}^{*}
\end{aligned}
$$

En posant $p \equiv \frac{P_{F}}{P_{H}}$ et $p^{*} \equiv \frac{P_{F}^{*}}{P_{H}^{*}}$, on a donc

$$
p^{*}=p(1-\tau)^{2}
$$

Les conditions du premier ordre impliquent

$$
\frac{C_{H}}{C_{F}}=p^{\theta} \text { et } \frac{C_{H}^{*}}{C_{F}^{*}}=\left(p^{*}\right)^{\theta}
$$

En combinant (28) et (29), on obtient

$$
\frac{C_{H}}{C_{F}}=(1-\tau)^{-2 \theta} \frac{C_{H}^{*}}{C_{F}^{*}}
$$

Sous l'hypothèse simplificatrice $Y_{H}=Y_{F}, \frac{C_{H}}{C_{F}}=\frac{C_{F}^{*}}{C_{H}^{*}}$ et l'équation (30) conduisent à

$$
\frac{C_{H}}{C_{F}}=\frac{C_{F}^{*}}{C_{H}^{*}}=(1-\tau)^{-\theta}=p^{\theta}
$$

de sorte que le rapport en valeur des consommation nationale et étrangère s'écrit

$$
\frac{C_{H}}{p C_{F}}=\frac{p^{*} C_{F}^{*}}{C_{H}^{*}}=(1-\tau)^{1-\theta}
$$

En l'absence de coûts de transport $(\tau=0), \frac{C_{H}}{p C_{F}}=1$ : le consommateur consomme des biens nationaux et étrangers en parts égales. En revanche, la présence de coûts de transport $(\tau>0)$ 
explique la préférence du consommateur pour le bien national. Pour une valeur suffisamment élevée de $\theta$, cette préférence nationale pourrait engendrer une forte volatilité des taux de change et une faible corrélation croisée des consommations. Les coûts de transport donnent lieu à une préférence nationale dans la consommation : le consommateur va préférer le bien qui ne subit pas de coût de transport. Ce faisant, la consommation nationale devient fortement liée aux fluctuations de la production locale, ce qui vient réduire la corrélation internationale des consommations.

Par ailleurs, comme nous l'avons souligné dans la section 3, la préférence du consommateur pour le bien national rend les paniers de consommation différents dans les 2 pays. La parité des pouvoir d'achat n'est donc plus vérifiée, le taux de change réel varie. Obstfeld \& Rogoff (2000b) insistent sur l'importance des coûts de transport dans les choix de consommation à l'échelle internationale et les fluctuations du taux de change réel. Or l'importance des coûts de transport constitue la caractéristique qui distingue un bien échangé d'un bien non échangé. L'article de Obstfeld \& Rogoff (2000b) invite donc les chercheurs à repenser cette distinction.

Des travaux empiriques remettent également en cause le manque d'intérêt pour la distinction entre biens échangeables et non échangeables. Betts \& Kehoe (2006) constatent que, depuis les travaux pionniers de Backus et al. (1992), les faits stylisés ne sont calculés qu'à partir des fluctuations du taux de change réel vis-à-vis des Etats-Unis. Betts \& Kehoe (2006) élargissent le champ des pays considérés et décomposent les fluctuations du taux de change dans 52 pays (soit 1326 taux de change réel bilatéraux) entre 1980 et 2000. Ils concluent que plus d'un tiers des fluctuations du taux de change réel $\Gamma$ sont dues aux variations du prix relatif des biens échangeables et non échangeables $\Gamma^{N}$.

Plusieurs travaux empiriques soulignent que la contribution du prix relatif des biens échangeables et non échangeables aux variations du taux de change réel peut atteindre 50 à $70 \%$.

Burstein et al. (2005) estiment que les conclusions d'Engel (1993, 1999) sont biaisées car les indices de prix à la consommation incluent les coûts de distribution qui sont par nature des éléments non échangeables. La part des fluctuations de $\Gamma^{N}$ dans $\Gamma$ serait donc surestimée. Lorsque le prix relatif des biens échangeables est mesuré correctement, soit purgé des coûts de distribution ${ }^{7}$, le prix relatif des biens échangeables et non échangeables explique la moitié des fluctuations du taux de change réel.

Parsley \& Wei (2006) critiquent également les résultats d'Engel $(1993,1999)$ en soulignant que la part des biens échangeables et non échangeables dans le panier de consommation (les paramètres $\xi$ et $\xi^{*}$ dans l'équation (27)) est mal estimée. Engel estime ces proportions à partir de données agrégées sur l'indice de prix à la consommation, susceptibles donc de fournir des mesures imprécises du poids des biens non échangeables dans l'économie. En revanche, sur

\footnotetext{
${ }^{7}$ Burstein et al. (2005) considèrent donc les prix au moment du déchargement du bien sur le dock, ce qui correspond au prix du bien échangé sans les coûts de distribution.
} 
l'indice Big Mac, indice fortement corrélé à l'indice de prix à la consommation, ces proportions sont connues avec exactitude. Dans Parsley \& Wei (2006), les indices de prix à la consommation dans les équations (24) et (25) sont remplacés par le prix du Big Mac mesuré dans 2 pays et les prix $p^{T}$ et $p^{N}$ mesurent les prix des biens échangeables et non échangeables entrant dans la composition du Big Mac. Il est possible de connaître avec exactitude la part des biens échangeables et non échangeables dans la composition du Big Mac. Parsley \& Wei (2006). Plus de $50 \%$ des fluctuations du taux de change réel (mesuré en prix relatif réel des Big Macs) est expliquée par le prix relatif des biens non échangeables.

Enfin, $70 \%$ des fluctuations du taux de change réel seraient affectées par le prix relatif des biens échangeables et non échangeables dans des pays émergents tel que le Mexique (Mendoza (2005)).

\subsection{Une distinction endogène entre biens échangés et non échangés}

Les travaux empiriques et théoriques viennent renouveler l'intérêt des chercheurs pour les biens non échangeables, réhabilitant ainsi le rôle des différentiels de productivité dans la fluctuation du taux de change réel (Harrod (1933), Balassa (1964), Samuelson (1964)).

Les contributions récentes ont pour originalité d'enrichir les modèles de macroéconomie ouverte d'éléments puisés dans les théories du commerce international. En effet, ces dernières analysent la décision des firmes de s'engager dans les échanges internationaux. Un bien peut donc être échangé ou non échangé, en fonction des chocs qui affectent les entreprises. Les contributions de Bergin \& Glick (2006) et Ghironi \& Melitz (2005) explorent cette voie. Dans les deux types de travaux, la distinction entre bien échangé et non échangé devient endogène. Les firmes sont caractérisées par des niveaux de productivité (Ghironi \& Melitz (2005)) ou des coûts de transports (Bergin \& Glick (2006)) différents. A la suite d'un choc, certaines vont trouver profitable d'exporter au lieu de vendre leur production sur le marché national. Le bien non échangé devient donc échangé. Un examen attentif de ces deux séries de travaux permettent de comprendre la richesse des modèles théoriques proposés. Notons que ces travaux retiennent l'hypothèses de prix flexibles.

Les travaux de Hummels (2001) démontrent la grande diversité des coûts de transport (barrières douanières, non douanières, coût de transport proprement dit, coûts de publicité et de distribution) selon les biens considérés. Par ailleurs, ces coûts de transport jouerait un rôle essentiel dans la décision d'une entreprise de s'engager dans la conquête d'un marché étranger. Ces conclusions justifient donc la modélisation de Bergin \& Glick (2006) fondée sur l'hypothèse qu'il existe un continuum de firmes caractérisées par des coûts de transport différents. A l'instar d'Obstfeld \& Rogoff (2000b), les coûts de transport sont résumés par des coûts "iceberg" :

$$
p_{i}=\frac{p^{*}}{1+\tau_{i}}
$$


avec $p^{*}$ le prix mondial normalisé à $1, p_{i}$ le prix d'exportation du bien $i$ et $\tau_{i}$ son coût de transport. Notons que, lorsque le coût de transport augmente, le prix d'exportation baisse.

De plus, l'indice de prix des biens échangés dépend positivement de la part des biens non échangés dans l'économie, notée $n$. En effet, lorsqu'une part croissante de la production nationale n'est pas exportée (i.e. il n'est plus profitable d'exporter des biens dont le coût de transport apparaît trop élevé compte tenu des conditions économiques, ce sont donc les biens pour lesquels le prix d'exportation étaient les plus bas), ces biens ne sont plus inclus dans l'indice de prix des biens échangés. L'indice de prix des biens échangés augmente avec $n$ car il est calculé sur un ensemble de biens échangés duquel on a soustrait les biens caractérisés par les prix d'exportations les plus bas.

A la suite d'un choc de demande favorable aux biens non échangeables, avec une part exogène des biens non échangés dans l'économie, ce choc donne lieu à une hausse de prix des biens non échangés. Avec $n$ endogène, la hausse du prix relatif des biens échangés est atténuée par une hausse de la part des firmes qui souhaitent profiter de cette conjoncture favorable sur le marché national, délaissant alors les exportations ( $n$ augmente). La modélisation de Bergin \& Glick (2006) permet donc de rendre compte d'une faible volatilité du prix relatif des biens échangeables et non échangeables. En revanche, la volatilité de la déviation à la loi du prix unique est très volatile, conformément aux données. En effet, l'indice de prix des biens échangés dépend positivement de $n$. Les fluctuations endogènes de $n$ amplifient les réponses de l'indice de prix des biens échangés, engendrant de fortes variations de la déviation à la loi du prix unique.

Ghironi \& Melitz (2005) s'appuient également sur les théories du commerce international pour étudier les décision d'exporter des entreprises. Ces dernières sont caractérisées dans des niveaux de productivités différents. Les firmes subissent un coût fixe d'entrée sur le marché national et étranger ainsi que des coûts de transports par unité de bien exporté. Seules les entreprises les plus productives exportent leur produit, les entreprises les moins productives vendent localement leur production. Les chocs agrégés sur la productivité, les coûts d'entrée ou les coûts de transport incitent les entreprises à entrer ou se retirer des marchés national et étranger, modifiant ainsi la composition du panier de consommation. Ghironi \& Melitz (2005) parviennent à reproduire une corrélation positive de la production ainsi qu'une forte volatilité du taux de change réel.

Les mécanismes économiques s'apparentent à ceux mis en avant par Harrod (1933), Balassa (1964) et Samuelson (1964). Le taux de change réel s'écrit en fonction des écarts de productivité entre les pays. Dans un cadre théorique simple avec le travail comme unique facteur de production, le taux de change réel est fonction du différentiel des coûts du travail dans les deux pays. L'évolution de cette variable dépendra des entrées et sorties de firmes sur les marchés national et étranger. En raison de la présence de coûts d'entrée sur les marchés, les entrées de firmes 
prennent du temps, évoluant dans le modèle comme un stock de capital.

A la suite d'un choc de productivité favorable dans le pays national, nous retrouvons dans un premier temps l'effet volume cohérent avec une corrélation internationale positive des productions et l'effet prix (ou effet termes de l'échange) qui donne lieu à une évolution asymétrique des productions nationale et étrangère.

- Par l'effet volume, la hausse de la demande de bien concerne les productions nationales et étrangères, créant une conjoncture favorable dans les deux pays.

- En revanche, la hausse de la productivité du travail incite les ménages à offrir davantage de travail, ce qui se traduit, à l'équilibre, par une dépréciation du prix relatif des coûts du travail et donc du taux de change réel. La dépréciation rend les produits nationaux peu onéreux par rapport aux biens étrangers et profite aux exportateurs nationaux au détriment des exportateurs étrangers. L'effet prix engendre une évolution opposée des productions nationales et étrangères.

Grâce à l'entrée graduelle de nouvelles firmes sur le marché national, cette dépréciation du taux de change réel est atténuée, voire inversée. L'augmentation progressive du nombre de producteurs nationaux pousse la demande de travail à la hausse, conduisant à un renchérissement du coût du travail. Le taux de change réel s'apprécie. Plus généralement, la prise en compte des variations dans les entrées et sorties sur les marchés vient amplifier l'impact des chocs sur les écarts de productivité et donc les fluctuations du taux de change réel. Ghironi \& Melitz (2005) parviennent à reproduire une corrélation positive de la production ainsi qu'une forte volatilité du taux de change réel, sans toutefois rendre compte du faible niveau de corrélation internationale des consommations.

\section{Conclusion}

La contribution pionnière de Backus et al. (1994) qui étend le modèle RBC de base à un cadre international s'est révélée incapable de rendre compte de l'ampleur de la synchronisation cyclique des PIB et des consommations observée dans les données ainsi que de la forte volatilité du taux de change. La volonté de proposer des modèles capables de résoudre ces énigmes empiriques a suscité une abondante littérature. Les modèles DGSE parviennent aujourd'hui à rendre compte de ces faits stylisés en explorant les conséquences de l'incomplétude de marché ou de structures productives multisectorielles. La contribution d'Obstfeld \& Rogoff (1995), intégrant concurrence monopolistique et rigidités nominales, s'impose comme un modèle de référence car offrant des fondements microéconomiques à une cadre keynésien en économie ouverte. Les travaux empiriques confirment la pertinence de ce choix.

Les contributions récentes de la macroéconomie internationale ouvrent ce champ de recherche à des théories connexes telles que le commerce international ou la finance. Nous avons choisi 
d'illustrer l'apport des théories du commerce international dans la compréhension des énigmes empiriques. Les modèles DGSE en économie ouverte apportent donc des éléments de réponse concernant les décisions d'exportation des entreprises et leurs conséquences sur les fluctuations internationales. Cette accent sur les choix de localisation des entreprises apparaît essentiel aujourd'hui.

La prise en compte des théories de choix de portefeuille a également donné lieu à des articles récents $^{8}$. Nous évoquerons quelques éléments qui suggèrent la richesse de ce champ de recherche. Lane \& Milesi - Ferretti (2003, 2006) ont mis en évidence l'explosion des flux financiers internationaux associé à l'accroissement sans précédent des détentions croisées de titres financiers internationaux (dette, action et investissements directs). Cette ouverture des portefeuilles financiers aux titres étrangers peut donner lieu à des transferts de richesse internationaux en cas de modifications des cours des devises ou des prix des actifs financiers. Prenons l'exemple des Etats-Unis. Dans ce pays, les dettes sont essentiellement libellées en dollars et $70 \%$ des titres étrangers détenus par les résidents américains sont libellés en monnaie étrangère. Une dépréciation du dollar américain ou un dynamisme boursier plus marqué en Europe qu'aux Etats-Unis implique un transfert de richesse favorable aux Etats-Unis. Gourinchas \& Rey (2005) rappellent que les inquiétudes concernant l'ampleur du déficit commercial américain deviennent alors injustifiée. En 2005, le Bureau of Economic Analysis estimait à 85\% du PIB (107\%) les actifs (les dettes) extérieurs des Etats-Unis. Une dépréciation de 10\% du dollar conduit à un transfert de $5.9 \%$ de PIB $(0.7 \times 0.85 \times 0.1)$ du reste du monde vers les Etats-Unis. Ce transfert serait tout à fait susceptible de couvrir un déficit commercial de l'ordre de 5.3\% de PIB observé en 2005. Ces éventuelles fortes variations du prix des actifs et leurs conséquences significatives sur la valeur des portefeuille sont appelés "valuation effects". La compréhension des choix de portefeuille prend donc une importance particulière étant donné l'ampleur de ces effets.

\footnotetext{
${ }^{8}$ Voir Benigno (2001), Cavallo \& Ghironi (2002, 2004), Iscan et al. (2005), Ghironi et al. (2005). Obstfeld \& Rogoff (2000b), Coeurdacier (2005), Engel \& Matsumoto (2006), Heathcote \& Perri (2004) et Kollmann (2006).
} 


\section{ANNEXES}

\section{A Les données}

Les données trimestrielles sont extraites des Compte Nationaux de la base de l'OCDE. L'échantillon s'étend de 1970 :1 à 1999 :4, à l'exception de la France (1970:1-1998 :4), l'Allemagne (1970:1-1994:4), l'Italie (1970:1-1998:3) et de l'agrégat représentatif de l'Europe des 15 (1970:1-1999:3).

Les données sur l'emploi proviennent de la base des Statistiques Financières Internationales du FMI (indice d'emploi, base 100 en 1990).

Le taux de change réel $\Gamma$ est calculé à partir des séries de taux de change nominal (1 dollar américain $=E$ unités de monnaie nationale) et d'indices de prix à la consommation (base 100 en 1990) issues des Principaux Indicateurs Economiques de l'OCDE. Les séries s'étendent de 1971 :2 à 1999 :4. L'échantillon débute en période de changes fixes, à la fin du système de Bretton Woods. Le taux de change réel du pays $i$ noté $\Gamma_{i}$ et exprimé en logarithme s'écrit

$$
\Gamma_{i}=e_{i}+p_{U S}-p_{i}
$$

où $p_{U S}\left(p_{i}\right)$ désigne l'indice de prix à la consommation des Etats-Unis (du pays $i$ ) en log et $e_{i}=\log (E)$.

\section{B Les caractéristiques cycliques nationales}

\section{B.1 Les faits stylisés}

Les tableaux (8) et (9) présentent les caractéristiques nationales des pays du G7 calculées entre 1970 :1 et 1999 :4. Plusieurs régularités empiriques apparaissent :

- l'investissement est deux à trois fois plus volatile que le produit tandis que la consommation ainsi que l'emploi sont caractérisés par des profils plus lisses que celui de la production.

- la production est très persistante.

Le trait distinctif d'une économie ouverte tient à sa capacité d'emprunter sur le marché international des fonds prêtables. Le comportement cyclique du solde commercial rapporté au produit, qui mesure la différence entre production et absorption, devrait refléter ces besoins de financement externe. Les propriétés de la balance commerciale résulte de deux forces : le désir des agents d'utiliser les marchés financiers internationaux pour lisser leur consommation et les mouvements érratiques de l'investissement qu'autorise une parfaite mobilité du capital. Nous observons, à l'instar de Backus et al. (1995), une légère contracyclicité du solde commercial (défini comme les exportations nettes des importations) rapporté au PIB (tableau 9). 
TAB. 8 - Caractéristiques nationales : volatilité

\begin{tabular}{|l|c|c|c|c|c|}
\hline & $\sigma$ en $\%$ & $\frac{\sigma .}{\sigma_{Y}}$ & \multicolumn{1}{c|}{ Volatilité } & \multicolumn{1}{l|}{ Relative } \\
\hline & $Y$ & $C$ & $I$ & $H$ & $\frac{N X}{Y}$ \\
\hline Canada & 1.52 & 0.95 & 2.86 & 1.29 & 1.01 \\
\hline France & 1.04 & 0.86 & 2.85 & 0.57 & 0.70 \\
\hline Allemagne & 1.50 & 0.88 & 2.66 & 1.23 & 0.52 \\
\hline Italie & 1.45 & 0.85 & 2.52 & 0.84 & 0.57 \\
\hline Japon & 1.53 & 0.91 & 2.39 & 0.76 & 0.40 \\
\hline Royaume-Uni & 1.66 & 1.11 & 2.28 & 0.82 & 0.50 \\
\hline Etats-Unis & 1.99 & 0.69 & 2.27 & 0.66 & 0.21 \\
\hline Europe des 15 & 1.02 & 0.84 & 2.49 & 1.18 & 0.37 \\
\hline
\end{tabular}

TAB. 9 - Caractéristiques nationales : procyclicité

\begin{tabular}{|c|c|c|c|c|c|}
\hline & Corrélation & avec & $\overline{Y_{t}}$ & & \\
\hline & $Y_{t-1}$ & $C_{t}$ & $I_{t}$ & $H_{t}$ & $\frac{N X_{t}}{Y_{t}}$ \\
\hline Canada & 0.85 & 0.67 & 0.73 & 0.64 & -0.11 \\
\hline France & 0.85 & 0.65 & 0.85 & 0.68 & -0.38 \\
\hline Allemagne & 0.87 & 0.75 & 0.87 & 0.63 & -0.13 \\
\hline Italie & 0.85 & 0.80 & 0.78 & 0.23 & -0.40 \\
\hline Japon & 0.83 & 0.81 & 0.93 & 0.22 & -0.47 \\
\hline Royaume-Uni & 0.82 & 0.81 & 0.67 & 0.60 & -0.29 \\
\hline Etats-Unis & 0.89 & 0.93 & 0.90 & 0.68 & -0.60 \\
\hline Europe des 15 & 0.86 & 0.83 & 0.90 & 0.64 & -0.44 \\
\hline
\end{tabular}

\section{B.2 Les simulations}

Le modèle fournit une bonne représentation des propriétés cycliques (tableau 10). En particulier, les trois faits stylisés mentionnés dans la section B.1 sont bien reproduits par le modèle à l'exception de la volatilité de la consommation. Cette dernière est trop lisse par rapport aux faits stylisés en raison du comportement intertemporel des agents.

TAB. 10 - Simulations

\begin{tabular}{|c|c|c|c|}
\hline & $\frac{\sigma .}{\sigma_{Y}}$ & $\rho(., Y)$ & $\mathrm{AR}(1)$ \\
\hline$Y$ & $1.38\left(\sigma_{Y}\right)$ & 1 & 0.67 \\
\hline$C^{C}$ & 0.30 & 0.84 & 0.77 \\
\hline$H$ & 0.61 & 0.98 & 0.66 \\
\hline$I^{C}$ & 3.33 & 0.96 & 0.65 \\
\hline$N X$ & 0.31 & -0.32 & 0.74 \\
\hline$\tau$ & 0.32 & 0.55 & 0.80 \\
\hline$\Gamma$ & 0.18 & 0.55 & 0.80 \\
\hline
\end{tabular}




\section{Résolution et simulation}

L'ensemble des conditions du premier ordre ainsi que les équilibres sur les marchés constituent le système non linéaire. Les équations statiques sont

$$
\begin{aligned}
\frac{1}{C_{i t}^{C}} & =\lambda_{i t} P_{i t}^{C} \quad i=1,2 \\
Y_{1 t}+\tau_{t} Y_{2 t} & =\sum_{i=1}^{2} P_{i}^{C}\left(C_{i t}^{C}+I_{i t}^{C}\right)+C A_{1 t}+\tau_{t} C A_{2 t} \\
q_{1 t} & =P_{1 t}^{C}+\phi\left(I_{1 t}^{C}-\delta K_{1 t}^{C}\right) \\
q_{2 t} & =P_{2 t}^{C}+\tau_{t} \phi\left(I_{2 t}^{C}-\delta K_{2 t}^{C}\right) \\
\frac{\gamma_{H}}{1-H_{1 t}} & =w_{1 t} \lambda_{1 t} \\
\frac{\gamma_{H}}{1-H_{2 t}} & =w_{2 t} \lambda_{2 t} \tau_{t} \\
Y_{i t} & =a_{i t}\left(K_{i t}^{C}\right)^{\alpha}\left(H_{i t}\right)^{1-\alpha} \quad i=1,2 \\
P_{1 t}^{C} & =\left[\gamma+(1-\gamma) \tau_{t}^{1-\theta}\right]^{\frac{1}{1-\theta}} \\
P_{2 t}^{C} & =\left[\gamma \tau_{t}^{1-\theta}+(1-\gamma)\right]^{\frac{1}{1-\theta}} \\
\hat{\Gamma}_{t} & =(2 \gamma-1) \hat{\tau}_{t}
\end{aligned}
$$

En outre, on a $\hat{\Gamma}_{t}=(2 \gamma-1) \hat{\tau}_{t}$

Les équations dynamiques sont

$$
\begin{aligned}
K_{i t+1}^{C} & =(1-\delta) K_{i t}^{C}+I_{i t}^{C} \quad i=1,2 \\
a_{i t+1} & =\rho_{a} a_{i t}+\varepsilon_{t} \quad i=1,2 \\
q_{1 t} & =\beta E_{t}\left[\frac{\lambda_{1 t+1}}{\lambda_{1 t}}\left(\alpha \frac{Y_{1 t+1}}{K_{1 t+1}^{C}}+q_{1 t+1}-\delta P_{1 t+1}^{C}\right)\right] \\
q_{2 t} & =\beta E_{t}\left[\frac{\lambda_{2 t+1}}{\lambda_{2 t}}\left(\tau_{t+1} \alpha \frac{Y_{2 t+1}}{K_{2 t+1}^{C}}+q_{2 t+1}-\delta P_{2 t+1}^{C}\right)\right]
\end{aligned}
$$

Les variables qui composent le système Etat-Mesure sont

- Les variables de contrôle

$$
C_{1 t}^{C}, C_{2 t}^{C}, I_{1 t}, I_{2 t}, H_{1 t}, H_{2 t}, Y_{1 t}, Y_{2 t}, P_{1 t}^{C}, P_{2 t}^{C}, \tau_{t}, \lambda_{t}
$$

- Les variables backward $K_{1 t}^{C}, K_{2 t}^{C}$

- Les chocs exogènes

$a_{1 t}, a_{2 t}$ 
- Les variables forward

$q_{1 t}, q_{2 t}$

La phase de validation du modèle s'effectue en deux étapes : une validation qualitative et une validation quantitative.

- Les fonctions de réponse constituent un moyen de valider qualitativement les mécanismes du modèle et reposent sur l'écriture espace-état des règles de décision. Les fonctions de réponse retracent la dynamique du modèle à la suite d'une hausse temporaire de $1 \% \mathrm{du}$ choc technologique. La réponse instantanée des variables backward est donnée par

$$
S_{B, t}=M_{S E} \times 1
$$

A l'horizon $j$, la réponse des variables backward est obtenue par

$$
S_{B, t+j}=M_{S S} \times S_{B, t+j-1}
$$

tandis que celle des variables de contrôle résulte de

$$
C_{t+j}=\left(M_{1}\right)^{-1} M_{2} Q S_{B, t+j}
$$

- Lorsque nous procédons aux simulations du modèle, nous tirons des vecteurs d'innovations $\left\{\varepsilon_{1 t}^{a}\right\}_{0 \preceq t \preceq T},\left\{\varepsilon_{2 t}^{a}\right\}_{0 \preceq t \preceq T} . T=120$ puisque cela correspond à la longueur des séries chronologiques à partir desquelles sont calculés les faits stylisés. Nous reconstruisons les variables d'intérêt dont la composante cyclique est identifiée par le filtre de Hodrick \& Prescott (1997). Les statistiques (écart-type, corrélations) sont ensuite calculées. L'opération est répétée 100 fois. Nous reportons dans les tableaux la moyenne pour chaque statistique obtenue sur les 100 simulations. 


\section{References}

Ambler, S., Cardia, E., \& Zimmermann, C. (2002). International transmission of the business cycle in a multi-sectoral model. European Economic Review, 46(2), 273-300.

Ambler, S., Cardia, E., \& Zimmermann, C. (2004). International business cycles : What are the facts? Journal of monetary economics, 51(290), 257-276.

Aschauer, D. (1985). Fiscal policy and aggregate demand. American Economic Review, 75, $117-127$.

Backus, D., Kehoe, P., \& Kydland, F. (1992). International real business cycles. Journal of Political Economy, 100(4), 745-775.

Backus, D., Kehoe, P., \& Kydland, F. (1994). Dynamics of the trade balance and the terms of trade : The j-curve? American Economic Review, 84(1), 84-103.

Backus, D., Kehoe, P., \& Kydland, F. (1995). International real business cycles : Theory versus evidence. In T. Cooley (Ed.), Frontiers of Business Cycle Research (pp. 213-231). Princeton University Press.

Balassa, B. (1964). The purchasing power parity doctrine : A reappraisal. Journal of Political Economy, 72, 584-596.

Barro, R. (1981). Output effects of government purchases. Journal of Political Economy, 81(6), $1086-1121$.

Baxter, M. \& Crucini, M. (1995). Business cycle and the asset structure of foreign trade. International Economic Review, 36(4), 821-854.

Bec, F. (1994). La transmission internationale des fluctuations : une explication de la corrélation croisée des consommations. Revue Economique, 45, 89-114.

Benigno, P. (2001). Price Stability with Imperfect Financial Integration. CEPR Discussion Paper 2854 .

Bergin, P. (2003). Putting the new open economy macroeconomics to a test. Journal of International Economics, 60(1), 3-34.

Bergin, P. (2006). How well can the new open economy macroeconomics explain the exchange rate and current account? Journal of International Money and Finance.

Bergin, P. \& Glick, R. (2006). A model of endogenous nontradability and its implications for the current account. Review of International Economics, Forthcoming.

Betts, C. \& Devereux, M. (1996). The exchange rate in a model of pricing-to-market. European Economic Review, 96(29), 1007-1021.

Betts, C. \& Kehoe, T. (2006). U.s. real exchange rate fluctuations and relative price fluctuations. Journal of Monetary Economics, 53(7), 1297-1326. 
Burstein, A., Eichenbaum, M., \& Rebelo, S. (2005). The Importance of Nontradable Goods' Prices in Cyclical Real Exchange Rate Fluctuations. NBER Working Paper 11699, NBER.

Cavallo, M. \& Ghironi, F. (2002). Net foreign assets and the exchange rate : Redux revived. Journal of Monetary Economics, 49, 1057-1097.

Cavallo, M. \& Ghironi, F. (2004). Net foreign assets and exchange rate dynamics. In J. Hairault \& T. Sopraseuth (Eds.), Exchange Rate Dynamics : Routeledge.

Chari, V., Kehoe, P., \& McGrattan, E. (2002). Can sticky price models generate volatile and persistent real exchange rates? Review of Economic Studies, 69(3), 533-563.

Christiano, L. \& Eichenbaum, M. (1992). Current real business cycle theories and aggregate labor market fluctuations. American Economic Review, 6(4), 1-9.

Coeurdacier, N. (2005). Do Trade Costs in Goods Market Lead to Home Bias in Equities? Mimeo, PSE.

Costello, D. \& Praschnik, J. (1993). Intermediate Goods and the Transmission of International Business Cycles. Mimeo, University of Western Ontario.

Craine, R. (1975). Investment, adjustment costs and uncertainty. International Economic Review, 16(3), 648-661.

Devereux, M., Gregory, A., \& Smith, G. (1992). Realistic cross-country consumption correlations in a two-country equilibrium business cycle model. Journal of International Money and Finance, 11, 3-16.

Engel, C. (1993). Real exchange rates and relative prices : An empirical investigation. Journal of Monetary Economics, 32, 35-50.

Engel, C. (1999). Accounting for us real exchange rate changes. Journal of Political Economy, $107(3), 507-538$.

Engel, C. \& Matsumoto, A. (2006). Portfolio Choice in a Monetary Open-Economy DSGE Model. NBER Working Paper 12214, NBER.

Engel, C. \& Rogers, J. (1996). How wide is the border? American Economic Review, 86(5), $1112-25$.

Engel, C. \& Rogers, J. (2001). Deviations from purchasing power parity : Causes and welfare costs. Journal of International Economics, 55(666), 29-57.

Farmer, R. (1993). The Macroeconomics of Self-fulfilling Prophecies. MIT Press, Cambridge,MA.

Feenstra, R. \& Kendall, J. (1997). Pass-through of exchange rates and purchasing power parity. Journal of International Economics, 43, 237-261.

Frenkel, J. \& Razin, A. (1987). Fiscal Policies and the World Economy : An Intertemporal Approach. Cambridge : MIT Press. 
Gagnon, J. \& Knetter, M. (1995). Markup adjustment and exchange rate fluctuations : Evidence from panel data on automobile exports. Journal of International Money and Finance, 14, $289-310$.

Ghironi, F., Lee, J., \& Rebucci, A. (2005). The Valuation Channel of External Adjustment. Mimeo, IMF.

Ghironi, F. \& Melitz, M. (2005). International trade and macroeconomic dynamics with heterogeneous firms. Quarterly Journal of Economics, CXX, 865-915.

Giovannini, A. (1988). Exchange rates and traded goods prices. Journal of International Economics, 24(1/2), 45-68.

Goldberg, P. \& Knetter, M. (1997). Goods prices and exchange rates : What have we learned? Journal of Economic Literature, XXXV, 1243-1272.

Gourinchas, P. \& Rey, H. (2005). International Financial Adjustment. NBER Working Paper 11155, NBER.

Greenwood, J., Hercowitz, Z., \& Huffman, G. (1988). Investment, capacity utilization and the real business cycle. American Economic Review, 78, 402-417.

Hairault, J. \& Sopraseuth, T. (2005). Exchange rate dynamics and monetary shocks : A reappraisal. Review of International Economics, 13(3), 576-596.

Hairault, J.-O. (1998). Static Labor Supply in a Two-Good Two-Country Model : Explaining International Comovements. Mimeo, EUREQua University of Paris I.

Harrod, R. (1933). International Economics. London : James Nisbet and Cambridge Univerity Press.

Hau, H. (2000). Exchange rate determination under factor price rigidities. Journal of International Economics, 50(2), 421-447.

Heathcote, J. \& Perri, F. (2004). The International Diversification Puzzle is Not as Bad as You Think. Mimeo, Federal Reserve Bank of Minneapolis.

Hodrick, R. \& Prescott, E. (1997). Post war us business cycles : an empirical investigation. Journal of Money, Credit and Banking, 29, 1-16.

Huang, K. \& Liu, Z. (2006). Business cycles with staggered prices and international trade in intermediate goods. Journal of Monetary Economics, forthcoming.

Hummels, D. (2001). Toward a Geography of Trade Costs. Working paper, Purdue University.

Isard, P. (1977). How far can we push the law of one price? American Economic Review, 67(5), 942-948.

Iscan, T., Ghironi, F., \& Rebucci, A. (2005). Net Foreign Asset Position and Consumption Dynamics in the International Economy. IMF Working Paper 05/82, IMF. 
Kehoe, P. J. \& Perri, F. (2002). International business cycle with endogenous incomplete markets. Econometrica, 70(3), 907-928.

Knetter, M. (1993). International comparisons of pricing-to-market behaviour. American Economic Review, 83, 473-486.

Kollman, R. (1996). Incomplete asset markets and the cross-country consumption correlation puzzle. Journal of Economic Dynamics and Control, 20, 945-961.

Kollmann, R. (2001). Explaning international comovements of output and asset returns : The role of money and nominal rigidities. Journal of Economic Dynamics and Control, 25, $1547-1583$.

Kollmann, R. (2006). International Portfolio Equilibrium and the Current Account. CEPR Discussion Paper DP5512, CEPR.

Lane, P. \& Milese Ferretti, G. (2006). A global perspective on external positions. In R. Clarida (Ed.), Gry Current Account Imbalances : Sustainability and Adjustment: The University of Chicago Press.

Lane, P. \& Milesi Ferretti, G. M. (2003). International financial integration. IMF Staff Paper, $50,82-113$.

Long, J. \& Plosser, E. (1983). Real business cycles. Journal of Political Economy, 91, 39-69.

Mendoza, E. (2005). Real Exchange Rate Volatility and the Price of Nontradables in SuddenStop-Prone Economies. NBER Working Paper 11691, NBER.

Mussa, M. (1986). Nominal exchange rate regimes and the behavior of real exchange rates : Evidence and implications. Carnegie-Rochester Conference Series on Public Policy, North Holland, 25, 117-213.

Obstfeld, M. (1982). Aggregate spending and the terms of trade : Is there a laursen-mertzler effect? Quarterly Journal of Economics, (pp. 251-270).

Obstfeld, M. \& Rogoff, K. (1995). Exchange rate dynamics redux. Journal of Political Economy, 103(3), 624-660.

Obstfeld, M. \& Rogoff, K. (2000a). New directions for stochastic open economy models. Journal of International Economics, 50, 117-153.

Obstfeld, M. \& Rogoff, K. (2000b). The six major puzzles in international macroeconomics : Is there a common cause? In B. Bernanke \& K. Rogoff (Eds.), N.B.E.R Macroeconomic Annual 2000 (pp. 339-390). Cambridge : MIT Press.

Parsley, D. \& Wei, S. (2006). A Prism Into the P.P.P. Puzzles : The Micro-Foundations of Big Mac Real Exchange Rates. NBER Working Paper 10074, NBER.

Sachs, J. (1981). The current account and macroeconomic adjustment in the 1970s. Brooking Papers of Economic Activity, 12, 201-268. 
Samuelson, P. (1964). Theoretical notes on trade problems. Review on Economics and Statistics, $46,145-164$.

Schmitt-Grohe, S. \& Uribe, M. (2003). Closing small open economy models. Journal of International Economics, 61, 163-185.

Smets, F. \& Wouters, R. (2005). Bayesian new neoclassical synthesis (NNS) models : Modern tools for central banks. Journal of the European Economic Association, 3(2), 422-433.

Solow, R. (1957). Technical change and the aggregate production function. Review of Economics and Statistics, 39(3), 312-320.

Stockman, A. \& Tesar, L. (1995). Tastes and technology in a two-country model of the business cycle : Explaining international comovements. American Economic Review, 85(1), 168-185.

Zimmermann, C. (1997). Internation real business cycles among heterogeneous countries. European Economic Review, 41, 319-335. 